\title{
La réglementation administrative et le code pénal mis en tableaux
}

\author{
Pierre-Étienne Will *
}

Les pages qui suivent sont consacrées à la mise en tableaux des informations dans un type particulier de guides pratiques publiés à l'intention des administrateurs locaux à la fin de l'empire ${ }^{1}$. Ces guides présentaient à leurs lecteurs sous une forme aisément consultable un type de matériau dont la maîtrise était en effet d'une importance extrême dans l'exercice quotidien de leurs fonctions, et conditionnait indirectement leur notation, donc leur carrière, un matériau en outre qui ne cessait avec le temps de croître en volume et en complexité : je veux parler de la législation pénale et administrative, dont on trouvait les textes originaux dans les éditions successives du Code pénal (le Da Qing lüli 大淸律例), avec ses lois principales $(l i u)$ et ses lois additionnelles $(l i)$ parfois improprement appelées "précédents », ainsi que dans les recueils de règlements publiés irrégulièrement par les ministères sous la rubrique des « règlements sur les sanctions administratives»(chufen zeli 處份則例). Ces derniers concer-

\footnotetext{
* Pierre-Étienne Will est Professeur au Collège de France.

${ }^{1}$ Cet article est basé sur une communication présentée au colloque «De l'image à l'action : la dynamique des représentations visuelles dans la culture intellectuelle et religieuse de la Chine », organisé dans le cadre des «European and North American Exchanges in East Asian Studies » au Collège de France, les 3-5 septembre 2001. Je remercie Jérôme Bourgon pour ses suggestions concernant divers points de droit.
} 


\section{Pierre-Etienne Will}

naient ce qu'on est convenu d'appeler la «discipline bureaucratique » (lizhi 吏治), autrement dit le déroulement ordonné des procédures prescrites par la réglementation, le respect des délais, l'intégrité et l'impartialité des administrateurs, le fait que chacun reste à sa place et que le personnel subalterne est tenu en main, etc. Or, le Code pénal et les recueils réglementaires étaient des publications massives et difficiles d'accès, et jamais vraiment à jour puisque, d'une édition à l'autre, la législation ne cessait de s'enrichir de nouveaux textes applicables dès leur promulgation.

Appliqué à de tels contenus, la présentation en tableaux ne fait son apparition qu'au XVIII ${ }^{\mathrm{e}}$ siècle, alors qu'il existait depuis longtemps déjà une législation pénale et administrative complexe : je n'en ai pour le moment pas rencontré d'exemple antérieur à 1734. Après cette date en revanche, et jusqu'à la fin du XIX ${ }^{\mathrm{e}}$ siècle, la publication d'ouvrages se présentant comme des séries de tableaux résumant le code et la réglementation devient tout à fait fréquente, qu'il s'agisse de nouvelles créations, de simples mises à jour ou de piratages sans vergogne. Ces ouvrages posent plusieurs questions intéressantes : sur leur forme, d'abord - sur la façon dont étaient conçus et dont fonctionnaient les tableaux dont ils étaient intégralement composés mais aussi sur la nature exacte de leur contenu, sur le public auquel ils s'adressaient, et sur leur histoire en tant que genre éditorial.

\section{1.}

C'est en fait de l'histoire du genre que je vais partir. La description succincte des titres rencontrés au cours de mes investigations, plus ou moins regroupés par "écoles » et considérés dans leur développement chronologique, me permettra en même temps de donner une idée du contenu des ouvrages, de voir par qui ils étaient composés et publiés, de mieux cerner le public auquel ils s'adressaient, et de recueillir au passage quelques éléments intéressants regardant l'histoire de l'édition en Chine. Je reviendrai ensuite plus en détail, et à partir d'exemples précis, sur les questions de forme, autrement dit sur la nature même des " tableaux » en tant 
Réglementation administrative et code pénal mis en tableaux

qu'outils de communication. Nous verrons alors en quoi les différents formats concevables s'imposaient en effet comme autant de solutions particulièrement bien adaptées au contenu décrit (une réglementation foisonnante) et au but recherché (la rendre facile d'accès).

\section{Dong Gongzhen, Shen Xintian et leur héritage}

Comme indiqué, le premier manuel administratif entièrement composé de tableaux semble dater de 1734 . Non seulement je n'ai pas trouvé mention d'exemples antérieurs, mais un auteur de l'époque note dûment qu'avant l'ouvrage en question il n'existait sur le marché qu'une «trentaine de tableaux périmés publiés par des libraires" (fangke jiutu sanshi yu 坊刻舊圖三十餘) ${ }^{2}$. Le titre du nouvel ouvrage - qui se compose, lui, d'une bonne centaine de tableaux, et dont l'auteur est un certain Dong Gongzhen 董公振 - indique clairement de quoi il retourne : Matériaux faciles à consulter pour les conseillers techniques financiers et juridiques (Qiangu xingming bianlan 錢穀刑名便覽), en deux juan. Il s'agit, en d'autres termes, de fournir des données aisément accessibles aux conseillers techniques (les « amis de tente», muyou 幕友, ou « secrétaires privés » en franglais sinologique) en charge des deux domaines de compétence les plus cruciaux au sein des cabinets personnels des fonctionnaires de l'administration territoriale : d'une part, la fiscalité, et en fait l'ensemble de la gestion économique; d'autre part, le droit et tout ce qui touche à l'administration de la justice.

À première vue, notons-le, un titre comme Qiangu xingming bianlan pourrait être traduit «Matériaux faciles à consulter sur la fiscalité et la justice ». Mais le qiangu et le xingming sont bien des personnages, que l'on retrouve dans les titres d'une quantité de manuels qui leur sont explicitement destinés. Comme le confirment tous les ouvrages similaires

2 Shen Xintian, préface originale au Mingfa zhizhang (cf. ci-dessous). L'expression fangke, qui signifie à peu près "publication commerciale », n'est jamais très flatteuse : elle suggère une réalisation bâclée et un souci exclusif du profit. 


\section{Pierre-Étienne Will}

que j'ai pu examiner, le public visé par les guides en forme de tableaux, dont le Qiangu xingming bianlan est le prototype, c'est bien en premier lieu celui des muyou, des experts engagés par les fonctionnaires en titre et travaillant pour eux. L'objectif était de leur fournir des outils de travail à l'aide desquels ils pussent identifier rapidement les données légales et réglementaires des problèmes auxquels étaient confrontés leurs patrons et leur proposer des solutions, avant d'aller éventuellement consulter plus avant la littérature spécialisée. Il est vrai que les fonctionnaires étaient eux aussi encouragés à se servir de ce genre de guide, et en général à ne pas laisser le monopole du savoir technique à leurs conseillers. C'est pourquoi d'ailleurs les préfaciers aiment à insister sur le fait que l'ouvrage dont ils parlent devrait toujours être placé «à la droite " du fonctionnaire, ou posé sur sa table pendant les audiences, à moins qu'ils n'affirment que ce devrait être une lecture requise aussi bien pour ceux qui occupent un poste (les fonctionnaires) que pour ceux qui travaillent dans un cabinet (les muyou $)^{3}$... Mais il n'en reste pas moins que les compilations de tableaux représentant la législation pénale et administrative, comme d'ailleurs d'autres types de manuels portant également sur les domaines du qiangu et du xingming, non seulement émanaient presque entièrement du milieu des conseillers techniques ${ }^{4}$, mais encore s'adressaient en premier lieu à ceux-ci.

Comparé à la plupart de ses successeurs, l'ouvrage de Dong Gongzhen est relativement court : le premier chapitre, qui porte sur les problèmes fiscaux et financiers (le domaine du qiangu), se compose de 45 tableaux, et il y en a 52 dans le second, consacré au domaine judiciaire.

\footnotetext{
${ }^{3}$ Ainsi la préface (1763) d'un certain Wu Shiying 吳士映 au Lüli tushuo, dont il sera question plus bas : « une lecture essentielle pour le fonctionnaire en poste comme pour les membres de son cabinet » (wei juguan rumu zhi yaolan 第居官入幕之要覽).

${ }^{4}$ Leurs auteurs sont presque toujours eux-mêmes des muyou: ainsi Dong Gongzhen, l'auteur du Qiangu xingming bianlan, et Shen Xintian, celui du Mingfa zhizhang (cf. infra), que l'on peut considérer (et qui semblent avoir été considérés) comme les pères fondateurs du genre. Notons au passage que les autres auteurs les désignent parfois par leurs $z i$ respectifs, Nanhou 南厚 et Gengyu 耕于.
} 
Mais il a eu, justement, une postérité importante. Outre qu'on en trouve des éditions augmentées dans les années qui suivent sa première parution ${ }^{5}$, le Qiangu xingming bianlan est en effet à l'origine d'une série de manuels comparables, s'inspirant explicitement de lui et publiés jusque dans la seconde moitié du XIX ${ }^{\mathrm{e}}$ siècle, dont l'histoire est tout à fait suggestive du développement non seulement du genre lui-même, mais aussi de la demande croissante à laquelle il répondait.

Le premier titre à mentionner dans cette série est le Guide pratique du droit pénal (Mingfa zhizhang 明法指掌), composé par un conseiller technique spécialisé dans les problèmes juridiques nommé Shen Xintian 沈辛田, dont nous savons que la première édition, semble-t-il perdue aujourd'hui, a été imprimée en 1740. La date était mal choisie : la même année paraissait une édition complètement remaniée du Code pénal des Qing (le Da Qing lüli), la première dans laquelle les «précédents » (li) étaient intégrés aux lois fondamentales (liu) avec le statut de lois additionnelles, mais révisables ${ }^{6}$. Or, c'était la matière du code, précisément, que Shen Xintian avait entrepris dès 1734 de résumer et mettre en page dans les «tableaux faciles à consulter» du Mingfa zhizhang (zhizhang, «comme qui l'aurait en paume » en vieux français : l'idée est à peu près la même que pour bianlan dans le titre précédent). Cette malheureuse coïncidence explique la parution d'une version révisée et augmentée de l'ouvrage, sous le titre Mingfa zhizhang zengding 增訂, dès 1743, l'année

5 Une autre édition du même «hall ", le Chengyi tang 誠意堂, porte en page de titre la mention «augmenté en 1737 »; une autre encore, de 1742, porte le titre Zengbu 增補 qiangu xingming bianlan et se présente comme une « nouvelle gravure »; elle comporte effectivement neuf tableaux supplémentaires dans le second chapitre.

${ }^{6}$ Sur cette mutation dans le format du code pénal, qui a eu des répercussions profondes sur la pensée et la pratique juridiques jusqu'à la fin des Qing, cf. Jérôme Bourgon, "L'émergence d'une communauté de juristes à la fin de l'empire », communication faite au Collège de France le 26 mars 2003. 


\section{Pierre-Étienne Will}

où Shen Xintian a été, nous dit-il, à même d'examiner en détail la nouvelle version du Code pénal promulguée en $1740^{7}$.

L'histoire de la publication du Mingfa zhizhang, telle qu'on peut la reconstituer à partir des préfaces et des instructions aux lecteurs (fanli), est instructive à plusieurs égards. Il y a d'abord le problème évoqué à l'instant, que l'on retrouve avec la plupart des ouvrages de ce type, de l'évolution du corpus des règlements et précédents: s'ils veulent être vendables, les ouvrages qui en proposent des adaptations ou des résumés sous quelque forme que ce soit doivent être révisés à intervalles réguliers, et c'est particulièrement nécessaire lorsque le gouvernement fait paraître une nouvelle édition du Code ou des Chufen zeli. Dans le cas de l'édition révisée du Mingfa zhizhang gravée en 1743, une note de Shen Xintian affirme que le nouveau code paru en 1740 renouvelait de 30 à 40 pour cent le contenu de la précédente mouture ${ }^{8}$ : c'est bien ce qui l'a contraint à faire regraver entièrement son ouvrage et à y ajouter une cinquantaine de tableaux (portant le total à 255 ) ; et la preuve qu'il s'agit effectivement d'une nouvelle édition, et non pas d'une version marginalement corrigée mais abusivement vendue, comme c'est si souvent le cas, comme une « nouvelle gravure» (en faisant précéder des mots xinke 新刻 ou xinjuan 新鋃 le titre sur la page de couverture), c'est que Shen Xintian dit expressément que les planches d'impression de l'ancienne édition peuvent désormais être rabotées et affectées à un autre usage.

Cela étant, la publication de nouvelles versions du Code pénal (une en théorie tous les cinq ans) ou des corpus de précédents des différents ministères ne faisait que récapituler à intervalles plus ou moins réguliers un processus continu de création législative dont la bureaucratie était tenue au courant par la Gazette de Pékin et par les circulaires dont l'inondait le

${ }^{7}$ Le fanli de cette édition révisée précise que les lois additionnelles postérieures à la parution du nouveau code et promulguées jusqu'à l'été 1743 ont également été intégrées aux tableaux.

${ }^{8}$ C'est-à-dire le $D a$ Qing lü jijie 大瑇律集解 (le Code et ses commentaires) dans son édition de 1725 . 
gouvernement central, et dont elle était supposée tenir compte au fur et à mesure. Des ouvrages comme le Qiangu xingming bianlan, le Mingfa zhizhang et d'autres du même type intègrent cette dimension de différentes façons : suivant les cas, les tirages successifs s'enrichissent de suppléments intercalés ${ }^{9}$, les corrections sont faites directement sur les planches d'impression, ou même, indépendamment de ces mises à jour (parfois signalées par un sceau imprimé sur la page de titre, précisant la date jusqu'à laquelle on a tenu compte des nouveaux règlements), le lecteur est invité à copier lui-même les textes entrés en vigueur depuis l'impression de l'exemplaire en sa possession en utilisant les espaces libres ou les pages blanches réservées par l'éditeur ${ }^{10}$.

La publication du Mingfa zhizhang est par ailleurs révélatrice de l'importance cruciale du droit pénal dans l'arsenal du savoir administratif, et tout d'abord du savoir qui était attendu des conseillers techniques et qui justifiait les salaires élevés auxquels ils pouvaient prétendre. Dans sa préface de 1740 Shen Xintian explique que ce qui l'a motivé à entreprendre dès 1734 la confection de tableaux résumant le code pénal, c'est la nécessité qui lui est apparue de remplacer les chapitres juridiques du Qiangu xingming bianlan de Dong Gongzhen, paru cette même année, par quelque chose de beaucoup plus complet. En effet, ce dont parlent avant tout les chapitres "xingming " du Qiangu xingming bianlan, ce sont les sanctions (chufen) encourues par les fonctionnaires en cas de mauvaise administration de la justice, et leur impact sur leur évaluation (kaocheng 考成) ${ }^{11}$; alors qu'aux yeux de Shen Xintian - du moins est-ce ce qu'il affirme - les problèmes judiciaires ressortissant au domaine du xingming (litt. les

${ }^{9}$ Certains exemplaires du Qiangu xingming bianlan que j'ai vus comportent des pages supplémentaires en intercalation, marquées you 又, citant des précédents postérieurs à 1734.

${ }^{10}$ Dans le Lüli tushuo zhangzhen, dont il sera question plus bas, chaque tableau tient sur le recto de la page, le verso étant laissé vide ; dans l'exemplaire que j'ai vu il porte en effet des ajouts manuscrits.

11 De fait l'ouvrage est parfois mentionné sous le titre Qiangu xingming chufen beilan : cf. Zengding Xingqian zhizhang (1744, décrit infra), fanli, deuxième paragraphe. 


\section{Pierre-Étienne Will}

« dénominations pénales », encore appelées zuiming 罪名) concernent en premier lieu la vie du peuple et mettent en cause un ensemble bien plus complexe de règlements et de tâches administratives.

Effectivement, l'apport de Shen Xintian a été d'introduire le code pénal dans le système de tableaux inventé par Dong Gongzhen et de réaliser une présentation graphique combinant les dénominations pénales - qui sont en fait le principe de classement dominant, puisque la majorité des tableaux portent en titre les types de crime énumérés dans le code -, les peines prévues par le code et les lois additionnelles, et les sanctions administratives s'appliquant en cas de faute imputable au fonctionnaire. Comme l'écrira le compilateur d'une recension beaucoup plus tardive de l'ouvrage : «Le travail de M. Shen avait pour principal objet d'[aider à] fixer les sentences et déterminer les dénominations légales; lorsque des sanctions administratives étaient également susceptibles de s'appliquer, elles aussi apparaissaient dans les différents tableaux 》 (沈氏著書專爲定罪 而設名法。有簡處份者, 附見各圖). La cohabitation parfois ambiguë et pas toujours facile de ces deux aspects - le droit criminel du code pénal et le droit administratif des chufen zeli, qui concerne bien plus que les seules fonctions judiciaires des administrateurs - caractérise une large part de la littérature dont il est question dans cet article. Certains ouvrages, pour reprendre les termes du même auteur, «considèrent les sanctions administratives et les dénominations pénales comme deux choses différentes et les traitent à part 》 (別載處份與刑名学二事), alors qu'en fait 《les sanctions administratives relèvent elles aussi de la loi » (處份亦法令也) ${ }^{12}$. Un autre auteur, Shi Zhongyin 石中隱, à qui l'on doit un ensemble important

${ }^{12}$ Cf. Xu Hao, préface au Mingda zhizhang tu (datée 1869). Xu Hao oppose ici la version originale du Mingfa zhizhang (qu'il appelle Mingfa zhizhang $t u$ ), dans laquelle les deux catégories étaient séparées, aux recensions du même ouvrage parues dans les années 1820 , où elles étaient synthétisées. Sans pouvoir entrer dans le détail, soulignons simplement que l'examen des tables des matières suggère qu'en réalité les choses ne sont pas aussi tranchées : les éditeurs successifs n'ont cessé de réarranger les matériaux de la façon qui leur paraissait la plus pratique pour l'utilisateur. 
de tableaux résumant les chufen zeli (dont je parlerai plus loin), note que ceux-ci contiennent une vaste quantité d'informations qui ne figurent pas dans le Code pénal et que les deux ensembles de règles sont l'un par rapport à l'autre comme «l'extérieur et l'intérieur » (biaoli 表裏).

Dans tous les cas, un certain nombre de publications ont cherché à faire la synthèse des efforts des deux inventeurs du genre, Shen Xintian (plutôt tourné vers les aspects judiciaires du gouvernement local) et son prédécesseur Dong Gongzhen (envisageant un plus large éventail d'activités et plutôt concerné par les conséquences des actions des fonctionnaires sur leurs propres carrières). Le Mingfa zhizhang, déjà, intègre comme on vient de le voir certains règlements des Chufen zeli dans ses tableaux. Les cinq tableaux de la première section de l'ouvrage (précédant la très longue section sur les homicides, par quoi débute la mise en tableaux du code pénal à proprement parler) sont d'ailleurs entièrement consacrés à un problème relevant purement de la discipline administrative : les « délais imposés » (xianqi 限期) auxquels les diverses autorités du bas en haut de la hiérarchie doivent se tenir pour procéder aux enquêtes et aux interrogatoires, et au-delà desquels les sanctions commencent à pleuvoir si l'on n'a pas réussi à tout boucler à temps. Cette section sur les délais imposés restera en tête de toutes les moutures postérieures de l'ouvrage, et elle ne cessera de se développer en taille et en précision.

En plus de ses deux juan, la version révisée de l'ouvrage parue en 1743 (le Mingfa zhizhang zending) comporte un fascicule en annexe intitulé Mingfa zhizhang zengding shangjuan bianlan 上卷便覽, consacré aux sanctions administratives, qui est en fait une version quelque peu révisée de la section « qiangu » du Qiangu xingming bianlan de Dong Gongzhen, dûment salué comme auteur d'origine. (La plupart des 72 tableaux qui la composent concernent en effet la fiscalité et les finances, même si certains portent sur d'autres aspects de l'administration.) Shen Xintian précise d'ailleurs dans une note la raison de cet emprunt : à l'époque où il composait la version révisée de son ouvrage, en 1743, il travaillait au service du juge provincial du Guangxi, et c'est parce que l'ouvrage de Dong Gongzhen était difficile à trouver dans les provinces frontalières que la maison 
d'édition qui s'apprêtait à graver le sien lui avait suggéré d'en reprendre la matière en annexe ; et c'est en effet ce qu'il avait fait, en se basant sur une copie manuscrite appartenant à un de ses collègues et en l'adaptant à son propre format ${ }^{13}$. Ce seul détail, soit dit en passant, nous montre que même les libraires opérant dans des régions éloignées des centres économiques et culturels de l'empire étaient au courant des travaux intéressants disponibles sur d'autres marchés et susceptibles d'être diffusés localement. De même remarquera-t-on l'importance des copies manuscrites des ouvrages, dont on trouve encore beaucoup d'exemplaires dans nos bibliothèques, et qui bien souvent servaient autant pour faire connaître un texte que ses éditions imprimées.

Quoi qu'il en soit, à peu près au même moment, en 1744, paraissait, non pas dans la province reculée du Guangxi mais à Pékin, un ouvrage intitulé Zengding xingqian zhizhang 增訂刑錢指掌, en 4 juan, conjointement attribué à nos deux auteurs. Les deux premiers chapitres reproduisent en effet les tableaux du Mingfa zhizhang de Shen Xintian (apparemment dans sa première version datant de 1740 , avec quelques amendements), et les deux derniers les tableaux de la partie «fiscale» du Qiangu xingming bianlan de Dong Gongzhen ${ }^{14}$. Contrairement aux titres précédemment cités, l'ouvrage est une publication authentiquement commerciale. Elle émane d'un de ces éditeurs de Liulichang - ici le Rongjin tang 榮錦堂 qui n'entreprenaient jamais rien sans être assurés d'avoir un marché, et qui faisaient leur publicité : comme pour d'autres du même genre, la couverture du Zengding xingqian zhizhang porte les caractères Shihuan yaolan 士宦要覽 (lecture indispensable à MM. les Fonctionnaires). De façon as-

${ }^{13} \mathrm{Cf}$. le colophon (ba) de Shen au bianlan annexé à son Mingfa zhizhang zengding. La maison d'édition doit être le Tongde tang 同德堂, qui a réalisé l'ouvrage et dont on suppose qu'il se trouvait à Guilin, la capitale du Guangxi.

${ }^{14} \mathrm{Ni}$ le préfixe zengding ("augmenté et révisé ») au début du titre ni l'indication xinjuan (« nouvelle gravure ») avant l'indication de la date n'impliquent nécessairement qu'il existait une édition antérieure du même ouvrage, ou, s'il en existait une, que la "nouvelle édition" est autre chose qu'une simple réimpression des mêmes planches: ces formules servent souvent d'argument publicitaire. 
sez remarquable d'ailleurs, le même ouvrage a été réimprimé plus d'un siècle après et sans la moindre modification par une autre maison de Liulichang, le Wenbao tang 文寶堂, en 1856 et à nouveau en 1868 , en format de poche!

Le statut des autres «halls " (tang) dont nous trouvons assez souvent la mention sur la page de titre des ouvrages n'est pas toujours clair : s'agit-il de libraires commerciaux, éventuellement subventionnés par des fonctionnaires, ou d'établissements philanthropiques sans but lucratif visant un public d' « hommes partageant le même idéal » (des tongren 同 人, ou même des tongzhi 同志) ? La distinction n'est pas toujours évidente. Le Tongde tang 同德堂, qui publie le Mingfa zhizhang zengding au Guangxi en 1743, porte comme bien d'autres un nom à résonance philanthropique ; cependant, ainsi qu'on vient de le voir, la maison avait aussi le sens du marché, et en tout état de cause nous savons que l'ouvrage n'a pu être publié que grâce aux encouragements et à l'aide financière du patron de Shen Xintian, le juge provincial Li Xiqin 李錫秦, auteur d'une des préfaces.

De fait, le rôle crucial de certains fonctionnaires, parfois de rang fort élevé, pour rendre possible la publication d'outils de travail élaborés par des conseillers techniques avec qui ils partagent le même intérêt, quand ce n'est la même passion, pour les problèmes d'administration, est souvent en évidence, ne serait-ce que dans les préfaces élogieuses dont ils ornent les ouvrages et qui servent certainement d'argument de vente. La première édition du Mingfa zhizhang n'avait pu paraître que grâce à la générosité d'un certain Chen Kefu 陳克復, préfet de Guangnan au Guangxi, qui avait accueilli Shen Xintian sur le chemin du retour après quelques années passées au service du juge provincial du Yunnan, et dont il nous est resté une préface : c'est avec son argent que l'ouvrage pourra être gravé, après une ultime mise au point par Shen et son frère - ils ajoutent encore une trentaine de tableaux -, dans leur sous-préfecture natale de Wucheng 烏城 (i.e., Huzhou, au Zhejiang). Et l'on vient de voir que la version révisée de 1743 doit son existence à un autre patron de Shen Xintian, Li Xiqin. 
La combinaison de hauts fonctionnaires, de muyou et de libraires intéressés que l'on vient d'évoquer est encore mieux en évidence dans la seconde partie de l'histoire du texte, curieusement séparée de la première par une période de près de quatre-vingts ans. Les ouvrages de Dong Gongzhen et Shen Xintian semblent avoir eu un certain succès, et comme nous le verrons ils répondaient en effet à un besoin réel. Shen Xintian, en tout cas, affirme dans sa préface de 1740 que cela fait longtemps que le Qiangu xingming bianlan de son collègue Dong « circule partout dans l'empire » ( 久已風行海內); quant à la première version de son propre Mingfa zhizhang (celle de 1740, justement), à en croire sa note de 1743 expliquant les raisons de la nouvelle version, «nombreux étaient les gens s'intéressant à leur métier qui en avaient un exemplaire sur leur table» (好事案頭多固一 帙) ${ }^{15}$. Les deux ouvrages ont eu des tirages successifs, intégrant à chaque fois les nouveautés de la réglementation, et comme on l'a vu la formule paraît avoir été suffisamment attrayante pour pousser un libraire de Liulichang - loin du Jiangnan et du Sud-Ouest, où nos deux auteurs exerçaient leur activité - à en publier une synthèse en 1744 .

Or, je n'ai pour le moment pas trouvé dans les bibliothèques de réédition ou de nouvelle mouture de ces deux ouvrages postérieure à $1745^{16}$, et jusqu'en 1824 ; et il faut croire qu'il n'en est effectivement pas paru, car Niu Dawei 紐大煒, le compilateur de cette nouvelle édition de 1824, qui porte le titre Mingfa zhizhang xinli zengding 名法指掌新例增訂 (en 4 juan, cette fois), affirme bien que le texte est resté en l'état depuis " plus de quatre-vingts ans », ce qui explique les révisions considérables auxquelles il a dû procéder. Une «majorité » des articles portant sur les crimes et les sanctions ont dû être complétés par d'autres, ou supprimés, ou modifiés, et l'ouvrage comporte désormais 360 tableaux au lieu des 325 de la recension de 1743 (en comptant les 70 de l'annexe empruntée à Dong Gongzhen); à

\footnotetext{
15 Mingfa zhizhang zengding, préface originale [1740] et note additionnelle (1743).

${ }^{16}$ La Bibliothèque Centrale de Taiwan possède une édition du Mingfa zhizhang zengding datant de 1745 et publiée à Hangzhou par une maison s'appelant le Youwen tang 有文堂, que je n'ai pu examiner.
} 
quoi s'ajoutent deux appendices dans le juan 4, consacrés respectivement aux «six biens mal acquis» (liuzang 六物, 21 tableaux) et aux sanctions administratives (gexiang chufen 各項處份, 53 tableaux). Il s'agit donc d'une recension fortement augmentée et remise à jour, même si c'est toujours le même ouvrage, avec les mêmes préfaces, les mêmes colophons, la même présentation, et le même nom ${ }^{17}$. Ce qui est en revanche nouveau, c'est qu'il s'agit aussi d'une recension «provincialisée », si l'on peut dire, au sens où, comme le spécifie le fanli, les règlements et précédents concernant spécifiquement le Guangdong ont été pris en compte, alors que le compilateur a laissé de côté ceux qui concernent la capitale ou telle autre province mais n'ont pas de domaine d'application au Guangdong. Ce genre de spécialisation provinciale se rencontre fréquemment dans les manuels compilés par les conseillers techniques et destinés à leurs propres collègues.

Mais contrairement à Dong Gongzhen et Shen Xintian, Niu Dawei n'est pas un conseiller technique : c'est un de ces fonctionnaires en attente de nomination, particulièrement nombreux au $\mathrm{XIX}^{\mathrm{e}}$ siècle, qui s'agglutinaient dans les capitales des provinces et travaillaient comme chargés de mission (weiyuan 委員) pour le compte des autorités provinciales en attendant, parfois fort longtemps, d'être titularisés dans une magistrature ${ }^{18}$. Or, le patron de Niu Dawei n'était autre que Ruan Yuan 阮元 (1764-1849), le fameux lettré et homme d'État du début du XIX ${ }^{\mathrm{e}}$ siècle, alors gouverneur général des deux Guang, qui l'avait donc chargé de

${ }^{17}$ Du moins sur la page de titre ; le titre complet, insérant les mots «xinli », apparaît dans les marges centrales des folios.

18 À partir d'une date que je n'ai pas réussi à déterminer, mais en tout cas pendant toute la durée du XIX ${ }^{\mathrm{e}}$ siècle, les nouveaux magistrats nommés par le ministère de la Fonction publique tiraient au sort une province, et non une localité particulière comme c'était le cas à l'origine (cf. mon étude «Creation, Conflict, and Routinization : The Appointment of Officials by Drawing Lots, 1594-1700», Ming Qing yanjiu, 2003, sous presse). Les « stagiaires " ainsi mis à la disposition des gouvernements provinciaux constituaient une force de travail administratif considérable et susceptible d'être affectée à toutes sortes de tâches ad hoc. 
préparer une version mise à jour et augmentée du vieil ouvrage de Shen Xintian. Cette nouvelle version, que l'utilisateur est invité à compléter lui-même au fur et à mesure de la parution de nouveaux règlements, est imprimée et vendue à Canton ${ }^{19}$. En sus de cette édition de 1824 on trouve une nouvelle impression de l'ouvrage, datée de 1860, exactement identique en dépit de la mention « augmenté et édité » (zengding) ${ }^{20}$.

Cette nouvelle recension de Niu Dawei n'est pas la seule parue pendant ces premières années du règne de Daoguang. En 1830 est imprimée, au Sichuan cette fois, une mouture un peu différente, mais elle aussi en 4 juan, intitulée Mingfa zhizhang xinzuan 新纂, dont le compilateur est un certain Huang Luxi 黃魯溪 ${ }^{21}$. Lui aussi est un magistrat en attente de nomination; mais pour refondre l'ancien travail de Shen Xintian et Dong Gongzhen il a bénéficié de l'aide d'un conseiller technique du nom de Shao Shengqing 邵繩淸 qui travaillait à son service alors qu'il effectuait une suppléance de magistrature à Xiaoxi 小溪 (Sichuan) en 1828-1829 - en fait il y a de fortes chances pour que Shao ait été l'auteur principal ${ }^{22}$. Le travail accompli à ce moment est repris avec l'aide de huit collaborateurs une fois Huang Luxi revenu à Chengdu pour reprendre sa situation de magistrat en attente de nomination; il est donc publié en 1830 , nous ne savons pas par quel libraire, mais certainement avec la bénédiction des plus

${ }^{19}$ Le lieu de vente est même précisé, ce qui est assez rare : la page de titre porte l'indication 《gravé au Guangdong et vendu au Yuan'an tang》奥東刊板遠安堂發凭.

${ }^{20}$ La section juridique (Law Library) de la Bibliothèque du Congrès possède (en deux exemplaires !) une autre édition encore, presque identique, mais non datée et visibiement tirée à partir d'une gravure différente, ou à tout le moins modifiée, notamment dans son système de pagination. J'en ai vu encore deux autres éditions, l'une d'une maison commerciale de Pékin, le Rongsheng tang 榮浾堂, datée de 1833, et une «augmentée 》 de 1907 qui semble en fait identique à toutes les autres.

21 Il en existe aussi une édition datée de 1866, apparemment identique.

22 Dans sa préface Huang Luxi présente simplement Shao comme son «pays » (wuxiang 吾鄕) : lui est de Wu xian, et Shao est de Changshu - ils sont donc tous les deux de Suzhou. Le fait qu'il l'employait comme muyou est confirmé par la préface du Dufa tucun (voir ci-dessous). 


\section{Réglementation administrative et code pénal mis en tableaux}

hautes autorités de la province puisque nous y trouvons, en plus de celle de Huang Luxi lui-même, des préfaces du gouverneur général (qui n'est autre que Qishan 琦善, cousin de l'empereur, dont les mésaventures diplomatiques bien connues appartiennent encore à l'avenir ${ }^{23}$, du trésorier provincial, du juge provincial, et de l'intendant de la gabelle et du thé ! (Toutes ces préfaces sont datées de 1830.) Il ne semble d'ailleurs pas qu'aucun de ces hauts personnages ait explicitement donné instruction à Huang, comme Ruan Yuan à Niu Dawei quelques années plus tôt, de produire un Mingfa zhizhang modernisé : dans sa propre préface Huang présente ce travail comme une sorte de passe-temps utile auquel il se serait livré avec son conseiller technique et compatriote lorsque sa suppléance de magistrature lui en laissait le loisir, et qu'il aurait ensuite montré à ses collègues de Chengdu (des magistrats en attente de nomination comme lui, peut-on supposer); ce sont eux qui non seulement l'auraient aidé à revoir son manuscrit (à le « collationner » 校正), mais auraient aussi contribué chacun de sa poche à l'impression. En bref, si l'ouvrage a en fin de compte reçu l'onction d'illustres préfaces, au départ c'était une opération d'administrateurs militants soucieux de mettre ce merveilleux instrument de travail à la disposition de tous ceux qui partagent leurs idéaux : yi gong tonghao 以公同好.

Comme avait déjà pu le constater Niu Dawei dans les années 1820 ou 1830, la mise à jour du travail admirable mais périmé de Shen Xintian supposait une substantielle refonte. À en croire la préface de Huang Luxi, au terme des quatre-vingts années écoulées depuis la dernière version de Shen Xintian, 60 à 70 pour cent des précédents ont été modifiés, et $20 \mathrm{ou}$ 30 pour cent des tableaux datant des années 1740 sont dépassés. Cette recension de Huang Luxi, qui affirme être à jour jusqu'en 1829 du point de vue du contenu, comporte une centaine de tableaux de moins que celle de Niu Dawei, mais elle est construite de façon analogue. Et de fait, on constate au détour d'une des préfaces que la recension de Niu Dawei a bien servi de modèle ; simplement, le contenu en a été vérifié et la présentation

\footnotetext{
${ }^{23}$ C'est lui qui a cédé Hong Kong aux Anglais en 1840 , et sa carrière en a subi un sérieux contrecoup.
} 
a été simplifiée, d'où le nombre moins élevé de tableaux. La preuve que toutes ces versions devaient se confondre plus ou moins dans la perception du public, c'est qu'on trouve du Mingfa zhizhang xinzuan une soi-disant « nouvelle édition » datée de 1832 (les planches d'impression sont conservées au Mianxing tang 勉行堂, mais il ne nous est pas précisé où cela se trouve), avec les mêmes prestigieuses préfaces, mais dont le texte proprement dit n'est rien d'autre que l'exacte reproduction de la recension de Niu Dawei, au moindre détail près et jusqu'au titre inscrit dans les marges centrales (i.e. Mingfa zhizhang xinli zengding). La seule différence, c'est qu'on a supprimé la note de Niu faisant suite au fanli et permettant effectivement de l'identifier comme compilateur! On a donc affaire à un piratage caractérisé, d'ailleurs réalisé de façon fort soigneuse, ce qui n'empêche bien sûr pas l'éditeur d'annoncer que les contrefaçons (fanke 翻刻) seront sévèrement poursuivies.

Huang Luxi, on vient de le voir, mentionne au passage l'aide qu'il a reçue de son conseiller Shao Shengqing lorsque le manuscrit du Mingfa zhizhang xinzuan a été mis au point en 1828 et 1829 . Or, six ans plus tard, en 1836, paraît une nouvelle mouture des tableaux originellement compilés par Dong Gongzhen et Shen Xintian, sous le titre, cette fois, de Tableaux extraits de la lecture du Code (Dufa tucun 讀法圖存), dont le compilateur n'est autre que le même Shao Shengqing ${ }^{24}$. La préface de l'ouvrage est due à un certain Liang Shijun 梁士俊, qui se présente comme un conseiller technique juriste, donc un collègue de Shao, dont il nous précise d'ailleurs qu'il exerçait cette spécialité depuis une trentaine d'années. Après avoir donc collaboré à la recension publiée par Huang Luxi, Sheng a été l'employé d'un certain Li Futang 李橎堂, un intendant ayant détenu pendant quelques temps les sceaux de juge provincial du Sichuan en tant que suppléant ; pendant cette période passée dans les locaux du premier tribu-

\footnotetext{
${ }^{24}$ En tête des chapitres Shao Shengqing est désigné comme « dessinateur-compilateur » (huibian 繪編); Huang Luxi (désigné par son $z i$, Xingchuan 杏川) continue d'apparaître, cette fois comme « superviseur 》 (jianding 鉒定).
} 
nal du Sichuan, Shao, a eu l'occasion d'analyser un grand nombre de cas. Le Dufa tucun est supposé distiller toute cette expérience.

C'est, à la date où il paraît, la recension la plus élaborée des tableaux de Dong et Shen. Le nombre de lois additionnelles et de règlements incorporés a été augmenté, de même que celui des sections (men 聞: il y en a 24 au lieu de 19 dans le Mingfa zhizhang xinzuan) et celui des tableaux (356 au lieu de 257) ; la répartition des tableaux a été occasionnellement modifiée (comme c'était d'ailleurs le cas pour chacune des recensions énumérées); la section sur la corruption et les détournements de fonds (liuzang) a été nettement étendue ; et le dernier juan comporte des ensembles nouveaux de tableaux portant sur les principes généraux du code pénal (i.e. la première partie, intitulée mingli 名例, précédant les six parties correspondant aux six grands domaines de l'administration) et sur les assises d'automne. On trouve aussi, comme d'ailleurs dans la recension signée par Huang Luxi, des annotations dans les marges supérieures renvoyant d'un tableau à l'autre lorsqu'on y trouve mentionnées des situations comparables. Ce procédé cherche à pallier l'une des principales difficultés présentées par le texte du code pénal pour ses utilisateurs, à savoir la dispersion des articles susceptibles d'être invoqués par analogie.

De façon intéressante, la recension de Shao Shengqing ne se prévaut pas du patronage de hauts personnages, contrairement à celle de Huang Luxi à laquelle il avait aussi participé. L'éditeur - c'est-à-dire le propriétaire des planches d'impression - n'est autre que la famille Shao à Yushan (虞山郡氏藏板), et non seulement l'adresse est donnée, mais aussi le prix pour obtenir un tirage, incluant le travail et les fournitures, et ce prix, 4 taels d'argent, est fort élevé. Mieux - et ce détail suggère un souci de rigueur assez inhabituel dans l'édition traditionnelle chinoise -, les exemplaires portent chacun un numéro de série : celui que j'ai pu consulter à l'Université Columbia est le $\mathrm{n}^{\circ} 189$.

Il existe de l'ouvrage une " édition mise à jour 》 datée de 1860, en tout point semblable à celle de 1836 , y compris pour l'indication de l'adresse et du prix. Nous avons vu tout à l'heure que la première recension réalisée au XIX ${ }^{\mathrm{e}}$ siècle - celle de Niu Dawei à Canton - a elle aussi été 
rééditée à l'identique en 1860 , à quelques additions mineures près ${ }^{25}$. Mais la saga éditoriale des tableaux conçus par Dong Gongzhen et Shen Xintian sous le règne de l'empereur Yongzheng ne s'arrête pas là. Le dernier avatar connu de l'ouvrage, portant le titre Mingfa zhizhang tu 圖, date de 1870 , et c'est cette fois une publication tout à fait officielle.

Le compilateur, $\mathrm{Xu}$ Hao 徐䫝, est en effet un préfet; comme l'indique sa préface, il a reçu instruction du gouverneur du Guangxi, Su Fengwen 蘇鳳文, de mettre au point une nouvelle version remise à jour en partant des recensions de Niu Dawei et Huang Luxi. (Il rappelle au passage que la version d'origine, le Mingfa zhizhang zengding de 1743, avait déjà été composée au Guangxi.) On trouve parmi les autres préfaciers un gouverneur général et deux gouverneurs ; l'ouvrage est imprimé et distribué par une de ces maisons d'édition semi-officielles fondées dans plusieurs capitales provinciales pendant la «restauration Tongzhi» avec l'encouragement actif de la cour ${ }^{26}$. Il s'agit en l'occurrence d'une des plus importantes et des plus actives, Hubei Chongwen shuju 湖北崇文書局, sise à Wuchang. En effet, le gouverneur du Hubei, à qui une copie du manuscrit avait été envoyée par son collègue du Guangxi, a décidé de publier l'ouvrage chez lui. En bref, cette ultime mouture d'une sorte de how-to book conçu quelque cent trente années plus tôt par de modestes conseillers techniques s'adressant essentiellement à leurs propres collègues est devenue un élément du programme de reconstruction politico-administrative promu par les hommes d'État les plus influents de l'empire au lendemain de l'écrasement des Taiping; et ses éditeurs et préfaciers se réclament explicitement de ces circonstances.

\footnotetext{
${ }^{25}$ Peut-être celles introduites dans une édition de 1846, dont j'ai vu un tirage mis à jour jusqu'en 1850 .

${ }^{26}$ Sur ces maisons semi-officielles et sur les éditions qu'elles produisaient (appelées juben 局本), voir Cao Zhi 曹之, Zhongguo guji banben xue 中國古籍版本學, Taipei : Hongye wenhua shiye youxian gongsi, 1994, p. 359-367 (édition originale : Wuhan : Wuhan daxue chubanshe, 1992); ainsi que Wang Zhonghan 王鐘翰, «Beijing changsi fangshu ji » 北京㸡寺訪書記, in id, Qingshi xinkao 清史新考 (Shenyang: Liaoning daxue chubanshe, 1997), p. 297-306, notamment p. 301.
} 
La mise à jour supervisée par Xu Hao apparaît tout à fait substantielle: elle prend en compte les précédents et lois additionnelles parus jusqu'en 1869 , élimine les règlements périmés, refond une partie des tableaux, crée de nouvelles sections, et réorganise en partie l'ouvrage. Surtout, elle confirme la vitalité et la popularité d'une formule éditoriale, d'une technique permettant de présenter de manière accessible une matière complexe mais cruciale pour les administrateurs ${ }^{27}$. Nous allons voir de suite - avant d'en examiner de plus près la logique - que Shen Xintian et ses continuateurs n'ont pas été les seuls à y recourir.

\section{Autres ouvrages}

Comme je l'ai souligné, il existe un curieux hiatus dans l'histoire éditoriale des recueils de tableaux tréés par Dong Gongzhen et Shen Xintian : huit décennies pendant lesquelles il n'en paraît aucune révision alors que par définition leur contenu appelait des révisions périodiques -, si bien que l'on en vient à se demander pourquoi c'est ce vieux modèle qui a été repris par divers auteurs au début du règne de Daoguang, alors qu'étaient parus entre temps d'autres ouvrages.

Je ne puis garantir que j'ai eu accès à la totalité des recueils de législation pénale et administrative mise en tableaux publiés aux $\mathrm{XVIII}^{\mathrm{e}}$ et $\mathrm{XIX}^{\mathrm{e}}$ siècles - ou pour être plus exact, à la totalité de ceux qui existent encore dans les bibliothèques; mais je crois néanmoins que les principaux d'entre eux me sont connus, et que par conséquent les quelques données qu'on trouvera ci-dessous couvrent l'essentiel du domaine. Je doute d'ailleurs que ce type d'ouvrage ait proliféré, en nombre de titres sinon en tirages (et en piratages), car la compilation, le dessin et la gravure de dizaines et de dizaines de tableaux parfois extrêmement compliqués, intégrant une matière elle-même complexe et que seuls les bons spécialistes pouvaient maîtriser, devaient représenter un investissement d'énergie et de

${ }^{27} 11$ existe une autre édition de la recension de Xu Hao, publiée en 1900 à Pékin par le Ronglu tang 榮錄堂. 


\section{Pierre-Étienne Will}

fonds assez considérable. C'est d'ailleurs la raison pour laquelle, lorsqu'une formule a du succès, on la conserve en se contentant de l'adapter au renouvellement de la législation ou d'y apporter des améliorations de détail, voire en la reproduisant telle quelle, même à des décennies de distance ; et il n'est pas indifférent de voir, à l'occasion, des publications rivales se critiquer l'une l'autre, comme si la concurrence était en effet sévère et limitée à un nombre restreint de publications.

Comme pour les ouvrages dont j'ai parlé jusqu'ici, les principaux auteurs du genre exerçaient le métier de conseiller technique ; et parfois il s'agissait non seulement de professionnels expérimentés, mais encore d'auteurs à qui leurs nombreux écrits spécialisés valaient une grande notoriété au sein de la bureaucratie, et que liait à des fonctionnaires parfois très haut placés une sorte de complicité professionnelle et d'admiration mutuelle dont on trouve l'expression dans les préfaces à ces écrits. Deux d'entre eux méritent avant tout d'être mentionnés, Wan Weihan 萬維翰 et Wang Youhuai 王又槐. Comme c'est si souvent le cas avec les lettrés ayant dû limiter leurs ambitions à une carrière de conseiller technique, nous n'avons qu'une idée vague, basée sur des indications glanées çà et là dans les préfaces de leurs ouvrages, de leur parcours et des fonctions qu'ils ont occupées, et nous ne connaissons même pas leurs dates précises de naissance et de mort. (Wan Weihan nous dit cependant être âgé de $71 s u i$ lorsqu'il signe un colophon daté de 1770.) Tout ce que nous savons dans le cas présent, c'est que Wan Weihan a été actif comme muyou au Zhili et au Zhejiang, notamment, au début et au milieu du règne de Qianlong (1736-1795), tandis que la plupart des ouvrages de Wang Youhuai sont parus dans les toutes dernières années de ce même règne (et donc du XVIII siècle), après une carrière de "vingt ou trente ans " comme conseiller technique spécialisé dans le droit ${ }^{28}$.

28 Outre les recueils de tableaux résumant le code et la réglementation qui sont décrits ci-dessous, l'on connait de Wan Weihan une introduction générale sur les fonctions de conseiller technique, le Muxue juyao 幕學舉要 (rédigé dans les années 1740, publié avec un colophon de l'auteur daté 1770); un manuel d'administration de la famine, le 
Réglementation administrative et code pénal mis en tableaux

\section{Wan Weihan et la concurrence}

Les Tableaux sur le Code pénal avec explications (Lüli tushuo 律 例圖說) de Wan Weihan, en 10 juan, ont connu de multiples tirages - j'en ai pour le moment identifié une dizaine -, couvrant apparemment la plus grande partie de la carrière de l'auteur : la préface de ce qui est visiblement la première édition date de 1750 , et le tirage le plus tardif dont j'aie connaissance ${ }^{29}$ a pu encore intégrer les nouveaux textes promulgués en 1784. L'ensemble de ces éditions proviennent du même lieu, le Yunhui tang 芸暉堂, c'est-à-dire la résidence de la famille Wan à Wujiang (Jiangsu), et il est clair qu'il s'agit là d'une entreprise de publication aussi sérieuse que « suivie" : les pages de titre portent la mention "les imitations seront poursuivies» (fanke bijiu 翻刻必究), et plusieurs des exemplaires que j'ai vus sont revêtus d'un sceau précisant la date jusqu'à laquelle le tirage en question a été mis à jour en tenant compte des derniers précédents parus. (Les mises à jour consistent généralement en tableaux ajoutés à la fin de chaque section, parfois en pages supplémentaires insérées ${ }^{30}$.) Il semble y avoir eu, essentiellement, trois éditions successives,

Huangzheng suoyan 荒政瑱言 (1752); un compendium d'informations sur la réglementation locale au Zhejiang, le Xingjian l $u$ 行簡錄 $(1770)$; une autre introduction aux fonctions de conseiller technique, le Xingqian zhinan 刑錢指南 (1773); un recueil des règlements provinciaux du Zhejiang, le Chenggui shiyi 成規拾遺 (1773); ainsi qu'une édition du code pénal avec toutes ses annotations, le Da Qing lü jizhu 大清律集 註. Wang Youhuai, quant à lui, a publié un manuel sur la procédure judiciaire, le Ban'an yaolüe 辦案要略; un manuel général à l'usage des conseillers techniques, le Xingqian bilan 刑錢必覽, (1793); un autre à l'usage des fiscalistes, le Qiangu beiyao 錢穀備要 (1793), ainsi qu'une édition critique avec commentaires du traité officiel de médecine légale, le Xiyuan lu jizheng 洗冤錄集證 (1796), qui devait servir de base à une multitude de publications analogues pendant toute la durée du $\mathrm{XIX}^{\mathrm{e}}$ siècle.

${ }^{29}$ Conservé ainsi que plusieurs autres au département juridique de la Bibliothèque du Congrès.

${ }^{30}$ L'un des tirages (appartenant à la collection de M. Tian Tao à Pékin) porte sur la page de titre un sceau indiquant: "Cet ouvrage est conservé dans la famille Wan; le texte 


\section{Pierre-Étienne Will}

avec à chaque fois des révisions systématiques, vers 1750 , vers 1763 , et en 1774 , ceci n'excluant pas, on vient de le voir, des tirages avec des mises à jour partielles.

L'ouvrage est en fait assez impressionnant. Il représente beaucoup plus qu'une simple mise en tableaux du code pénal et des sanctions administratives, même s'il fait aussi cela (et que par conséquent, comme il est dit dans le fanli de la première édition, on a "réuni les deux ouvrages

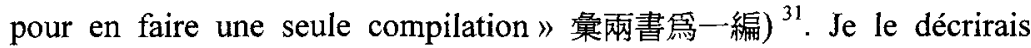
plutôt comme une sorte d'encyclopédie administrative mise en tableaux plus de 460 dans la première édition -, respectant l'ordre général du code (qui est aussi celui des six domaines traditionnels du gouvernement) sans s'interdire certains déplacements lorsque cela rend les choses plus logiques ${ }^{32}$, intégrant effectivement les lois et les lois additionnelles du code pénal et les sanctions administratives (chufen zeli) - ces dernières nombreuses surtout dans les sections sur la fonction publique (li) et sur les finances $(h u)$, alors que les articles du code dominent dans les quatre juan de la section sur les peines (xing) -, mais en les replaçant dans ce que je serais tenté d'appeler une présentation positive de la réglementation : comment les choses sont organisées et sont supposées se faire, et non pas seulement ce qu'on encourt si l'on enfreint telle ou telle règle. C'est ainsi que de nombreux tableaux commencent par exposer les règles, ou même par donner des définitions, un peu comme dans les recueils officiels du type Huidian, et n'entrent qu'en second lieu - c'est-à-dire plus bas dans le

original a fait l'objet d'ajouts ou de suppressions suivant les circonstances 》 (是集萬氏家 藏。原本隨時增減).

31 Il est cependant précisé que seule une sélection de règlements des chufen zeli, absents du texte du Code pénal, ont été intégrés - mais cela fait visiblement une masse imposante de matériaux.

32 «On les a arrangées par catégories, plutôt que de se conformer à l'ordre des lois [dans le code]" (比類敘之, 不以律爲次第也) (fanli de l'édition 1750). De même certains tableaux ont-ils été regroupés pour comparaison (以資參定). 
tableau - dans le détail des sanctions pour erreurs ou manquements ; certains même ne comportent aucune indication de sanction.

L'autre caractéristique de cette encyclopédie pour administrateurs, qui découle de sa présentation même (i.e. sous forme de tableaux), c'est bien sûr sa concision, l'absence revendiquée de tout commentaire personnel ou de tout développement, ainsi que l'absence d'indication de source et de date pour les lois additionnelles et les règlements. Les renseignements essentiels, mais précis, exprimés de la façon la plus neutre et la plus factuelle possible - incompressible, pourrait-on dire ${ }^{33}$-, sont regroupés de façon compacte et, autant que faire se peut, logique au sein de tableaux eux-mêmes arrangés de manière logique et, en tout cas, faciles à trouver, que ce soit en consultant les tables des matières détaillées qui précèdent chaque juan ou en repérant les titres dans les marges centrales, qui rendent chaque fascicule facile à feuilleter. Il est évident qu'une pareille mine de renseignements, arrangés de manière aussi lisible, était d'une aide précieuse pour les administrateurs non chevronnés comme pour ceux qui les assistaient dans leur travail quotidien. Il en va d'ailleurs de même pour l'historien d'aujourd'hui qui, bien plus encore que les bureaucrates de l'époque des Qing, a vite fait de se noyer dans les détails et les subtilités du Huidian shili, des Chufen zeli ou du Code pénal. Le Lüli tushuo mériterait assurément une bonne édition moderne !

Pour en finir avec Wan Weihan, j'ajouterai encore que parmi les différentes moutures de son texte on en trouve une qui porte le titre Lüli tushuo bian'e 辨誂, “avec discussion des erreurs 》, qui fait semble-t-il son apparition en 1762 ou 1763. Les erreurs en question sont consignées dans une liste à la fin de l'ouvrage, et ce sont celles non pas de Wan Weihan (il ne s'agit pas d'un errata), mais d'un certain Lu Weitian 魯緯天, un autre muyou juriste, originaire, lui, du Zhexi, et auteur d'un traité de toute

33 《Vraiment simple et impossible à abréger » (實簡而該非略), dit le fanli. 


\section{Pierre-Étienne Will}

évidence concurrent, jusque dans son titre : Lüli tushuo zhangzhen 掌珍, que j'hésite quand même à traduire par « votre Lüli tushuo préféré » ${ }^{34}$.

Le seul exemplaire de cet ouvrage que j'ai pu examiner ${ }^{35}$ est une édition de 1761, probablement la première parue sous ce titre: cela expliquerait que Wan Weihan ait réagi dès l'année suivante, comme le suggèrent les dates des préfaces de son bian'e, et surtout comme l'indique clairement sa propre préface de 1763 à une nouvelle édition du Lüli tushuo. 11 y accuse carrément la concurrence d'avoir piraté son propre travail : «Dans une publication commerciale que j'ai vue récemment, plus de cent tableaux [extraits de mon travail] ont été piratés sans vergogne et intégrés à un ouvrage existant, le Xingqian zhangzhen, qu'on a rebaptisé Lüli tushuo comme le mien, ceci dans le seul but de faire du profit" (近見坊刻 貿然剽取百餘圖, 列入掌珍鹤本, 亦號律例圖說, 以爲射利之計). De fait, les deux préfaces de l'édition 1761 du Lüli tushuo zhangzhen, qui sont datées de 1760, sont l'une et l'autre intitulées " préface au Xingqian zhangzhen 》 (刑錢掌珍序) - l'《 ouvrage existant》 en question. Mais, ajoute Wan Weihan, agir ainsi c'est tromper les gens, car les précédents évoluent et ses concurrents ont piraté les tableaux avec les anciens précédents, alors que lui, dans sa nouvelle édition, a supprimé tous les règlements périmés et a tenu compte des dernières publications; les éditions de son Lüli tushuo augmentées de la liste d'《identification des erreurs 》 (bian'e) enfoncent bien sûr le clou : vingt endroits contenant des erreurs sont signalés, étant bien précisé que ce n'est là qu'un échantillon ${ }^{36}$.

34 Zhangzhen, le « joyau dans la paume », se dit des personnes (notamment des enfants) objets d'un amour exclusif.

35 Je l'ai vu à la bibliothèque de l'Université Columbia il y a quelques années, mais il semble avoir été perdu depuis.

${ }^{36}$ C'est aussi pour se distinguer des contrefaçons (yanben 但本) vendues par les libraires que plusieurs tirages de l'ouvrage portent le titre Lüli tushuo zhengbian 正編. : cf. la "préface au Lüli tushuo zhengbian 》 de Shen Yi 沈翼 (1774). De son côté, le fanli du Lüli tushuo zhangzhen, qui rappelle dans son premier article que l'origine du genre remonte à l'ouvrage de Dong Gongzhen, y affirme aussi que la publication se justifie par le 
Quoi qu'il en soit de ces accusations, il se trouve que l'édition 1761 du Lüli tushuo zhangzhen est dite du Renji tang 忍濟堂 et que le fanli est signé par un certain Renji zhuren 忍濟主人, qui semble n'être autre que le compilateur, Lu Weitian (c'est ce que suggèrent les sceaux accompagnant la signature). Ce dernier, en d'autres termes, serait une sorte de clone de sa «victime », Wan Weihan : un muyou juriste compilant le même genre d'ouvrage ${ }^{37}$ et le publiant semblablement sous le sceau du « hall » familial. Nous sommes certes obligés de nous contenter d'aperçus furtifs sur les rivalités commerciales, les emprunts plus ou moins avoués et les manœuvres plus ou moins loyales qu'impliquaient de telles situations; mais n'oublions pas qu'il s'agissait après tout d'un très petit monde, d'un milieu professionnel composé de spécialistes et même d'activistes de l'administration, de statut extrêmement varié (ce que j'ai appelé ailleurs une «élite bureaucratique »), à l'intérieur duquel circulaient les individus comme les informations et les textes, et dont les membres étaient au courant de leurs travaux respectifs - sans parler bien sûr des libraires, dans les provinces et à Pékin, à l'affût de titres susceptibles de bien marcher. Une bonne partie des connexions entre les personnes concernées nous échappent, mais certaines apparaissent malgré tout au détour d'une préface ou peuvent être imaginées à partir de diverses indications. Il est par exemple impossible de ne pas remarquer que le même Li Xiqin, dont nous avons vu qu'il avait financé la publication du Mingfa zhizhang zengding en 1743 alors qu'il était juge provincial du Guangxi, donne également une préface à la première édition du Lüli tushuo en 1750 (il est vrai que Wan Weihan est un cousin du côté de sa mère) : il est à présent trésorier provincial du Guangxi, et si cette fois il ne semble pas avoir contribué financièrement, il

côté obsolète des ouvrages similaires en existence, parmi lesquels il mentionne, justement, le Lüli tushuo de Wan Weihan...

${ }^{37}$ Même taille approximativement (10 fascicules), même organisation des tableaux en six domaines (ou «six ministères 》), même combinaison de règlements extraits du Code pénal et des Chufen zeli. La principale différence peut-être est que la compilation de Lu Weitian a une orientation provinciale : en plus des lois applicables dans tout l'empire elle présente les règlements provinciaux applicables au Fujian. 
affirme au moins avoir vivement encouragé son jeune cousin à « confier au graveur » le manuscrit du Lüli tushuo qu'il lui a montré.

Le grand argument dans la polémique de Wan Weihan et de ses préfaciers contre les contrefaçons, c'est la nécessité d'être constamment au fait des derniers développements de la réglementation - d'où ces tirages remis à jour ou ces éditions complètement refondues qui se succèdent, ou au minimum ces espaces réservés où les usagers peuvent recopier les précédents postérieurs au tirage de l'ouvrage en leur possession. Tout à leur souci de gagner facilement de l'argent, les imitateurs, eux, ne seraient pas assez sérieux pour tenir compte du problème et se contenteraient de recopier des vieux tableaux. Or, un Lu Weitian était certainement conscient de la nécessité de prévoir des mises à jour, et dans le Lüli tushuo zhangzhen une page en blanc est réservée au dos de chaque tableau à cet effet. L'exemplaire que j'ai vu à l'Université Columbia porte plusieurs additions manuscrites dans ces pages, et en outre il est soigneusement ponctué en rouge, ce qui montre que cet ouvrage périmé et dangereux avait ses adeptes ! Nous verrons d'ailleurs plus loin à partir d'un exemple précis que le même tableau est plutôt mieux présenté chez $\mathrm{Lu}$ Weitian que chez Wan Weihan, ce qui suggérerait que, si plagiat il y a eu, c'était au moins un plagiat intelligent.

\section{Shi Zhongyin et Wang Youhuai}

Nous retrouvons avec l'histoire éditoriale d'un autre ouvrage mettant la réglementation en tableaux - celui-là exclusivement consacré aux sanctions administratives - le même souci d'être au dernier cri d'une législation dont à vrai dire peu de gens étaient capables de maîtriser les subtilités, les modifications incessantes et les contradictions qui en résultaient; nous retrouvons aussi ces mêmes coüncidences malheureuses, comme lorsqu'une nouvelle version du Code ou des Chufen zeli survient juste après la publication d'un ouvrage basé sur l'ancienne version. Il s'agit en l'occurrence des Tableaux fournissant l'essentiel sur les sanctions administratives (Zeli tuyao 則例圖要), compilés par un certain Shi 
Zhongyin 石中隱 en 1776, un an seulement avant la publication d'une nouvelle édition officielle des Chufen zeli, puis révisés et augmentés plusieurs fois pour tenir compte de cette nouvelle édition et de tous les règlements parus ensuite. L'ouvrage est publié dans une nouvelle gravure à Hangzhou en 1790 sous le titre Zeli tuyao bianlan 便覽, en 49 courts juan, et encore une fois en 1792 sous le titre, cette fois, Zengding 增訂 Zeli tuyao bianlan ${ }^{38}$. C'est cette dernière version qui fait intervenir Wang Youhuai, le conseiller technique auteur de nombreux ouvrages, et surtout extrêmement connu dans le milieu des administrateurs, auquel j'ai fait allusion plus haut. Dans sa préface de 1792, Wang Youhuai explique en effet que, dans ses déplacements d'un poste de conseiller technique à l'autre, il ne se séparait jamais de l'ouvrage de Shi Zhongyin, ce qui déjà est une bonne illustration du rôle d'alter ego tenu par les conseillers techniques par rapport à leurs employeurs : les sanctions administratives des chufen zeli visent en effet les fonctionnaires en titre, et non leurs conseillers, lesquels n'ont aucune existence officielle. Mais c'est aux conseillers qu'il incombe de prévoir les conséquences légales pour leur patron de décisions que bien souvent ils leur ont également suggérées, si bien que, si un ouvrage comme le Zeli tuyao et ses séquelles se présente explicitement comme destiné aux fonctionnaires débutants, ce sont en fait les conseillers des fonctionnaires qui s'en servent quotidiennement, même lorsque, comme Wang Youhuai, ils sont loin d'être eux-mêmes des débutants.

Peu importent ici les détails du travail - d'ailleurs limité - de révision et de réorganisation opéré par Wang Youhuai avec la collaboration d'un de ses « camarades » (tongzhi 同志, signifiant en l'occurrence qu'ils partagent le même idéal de qualité administrative), un certain Chen Jingxiu 陳敬修, ainsi que les lacunes qui subsistaient encore, affirme-t-il, dans le travail de Shi Zhongyin. Ce qui mérite en revanche d'être remarqué, c'est à quel point le marché semble avoir été porteur à la fin du XVIII ${ }^{\mathrm{e}}$ siècle pour

\footnotetext{
38 Je n'ai eu accès qu'à ces deux dernières moutures, mais l'histoire antérieure du texte est rapportée dans la préface rédigée par Shi Zhongyin en 1790.
} 


\section{Pierre-Étienne Will}

ce genre d'ouvrage. Comme nous l'avons vu, la grande compilation de Wan Weihan est encore remise à jour et imprimée au milieu des années 1780 (peut-être a-t-elle continué de l'être par la suite, mais pour le moment je n'en ai pas trouvé d'exemple). Wang Youhuai, pour sa part, mobilise au début des années 1790 ses considérables compétences pour publier une mouture améliorée d'une mise en tableaux des chufen zeli extrêmement massive et détaillée - le Zeli tuyao dont il vient d'être question -, dont on ne doute pas que la diffusion a bénéficié de son prestige. Enfin, troisième exemple que je voudrais citer ici, nous avons une mise en tableaux tout aussi massive du code pénal, cette fois - le code «pur», et non pas présenté en combinaison avec la réglementation administrative et les précédents disciplinaires -, parue sous le titre Lübiao 律表 (Le Code en tableaux), dont la première édition doit dater de 1778 approximativement et dont il existe des versions remises à jour et prenant en compte les précédents édictés jusqu'en $1788^{39}$.

\section{Le Lübiao}

Comme le Zeli tuyao bianlan, le Lübiao est un ouvrage d'un certain « poids » : il compte 38 juan (plus un juan d'introduction) ${ }^{40}$, relativement

\footnotetext{
39 Je n'ai pu examiner pour le moment la totalité des cinq (ou peut-être six) différentes éditions mentionnées dans divers catalogues de bibliothèques. Parmi celles que j'ai vues, l'une, non datée, qui pourrait être la première, a une préface précisant que les lois additionnelles des éditions du code pénal publiées en 1772 et 1778 ont été prises en compte. Une autre en format de poche (le titre est Xiuzhen Lübiao 袖珍律表) existe en deux versions publiées par deux libraires différents de Pékin, le Jindong shuhang 金東書 行 (datée de l'année bingzi 丙子, 1816 ou plus probablement 1876) et le Chengyu tang 承裕堂 (non datée), l'une et l'autre avec une préface de 1791 et comportant un fascicule séparé avec les lois additionnelles parues entre 1778 et 1788. Le même contenu se retrouve à l'identique dans une édition en grand format imprimée au Guizhou en 1883 .

${ }^{40}$ Les deux demiers juan sont en fait occupés par les «précédents sur l'arrestation des criminels " (dubu zeli 督捕則(例), qui ne font pas partie du code proprement dit; dans
} 
courts mais totalisant malgré tout cinq ou sept fascicules suivant les éditions, à quoi s'ajoute un fascicule supplémentaire proposant une mise en tableaux du fameux traité de médecine légale, le Xiyuan lu 洗冤錄, dans sa recension officielle de $1694^{41}$. La totalité des lois et des lois additionnelles est prise en compte, dans l'ordre du texte original du Code, de même que les notions générales (degrés de deuil, « huit caractères》 八字, six types de «biens mal acquis» 六䁍, etc.) dans le juan d'introduction, et les principaux commentaires sont mis à contribution lorsque c'est nécessaire, sous forme de notes dans les marges supérieures.

Zeng Hengde 曾恒德, l'auteur du Lübiao, n'est pas un conseiller technique, mais un fonctionnaire appartenant à l'une des seules administrations de l'empire où l'on trouvait de véritables spécialistes, comparables aux muyou par leur maîtrise de la «science du Code", et qui y faisaient souvent une grande partie de leur carrière: le ministère de la Justice (xingbu 刑部), où Zeng avait le rang de secrétaire (langzhong 郎中) et où il occupait les fonctions de compilateur au Bureau du code pénal (Lüli guan zuanxiu guan 律例館纂修官) ${ }^{42}$. On peut donc imaginer qu'il possédait une connaissance de première main du matériau qu'il lui avait fallu huit années de travail, affirme-t-il dans son fanli, pour dessiner et organiser lui-même sous forme de tableaux.

l'édition «de poche » ils sont comptés à part (le texte proprement dit n'a que 36 juan) et figurent dans un fascicule séparé.

${ }^{41}$ Ce fascicule supplémentaire a pour titre Xiyuan lu biao. Le Lüli tushuo de Wan Weihan inclut de même quelques tableaux résumant le Xiyuan lu. Depuis la promulgation d'un texte officiel du Xiyuan lu en 1694, l'ouvrage est plus ou moins considéré comme un appendice du code pénal, avec lequel il est souvent imprimé.

${ }^{42}$ Comme on sait, il n'y avait pas de conseillers techniques (muyou) dans les administrations métropolitaines. À en croire d'innombrables textes, le savoir spécialisé était monopolisé par les commis des bureaux ministériels (les fameux $l i$ 更, dont certains auteurs au $\mathrm{XIX}^{\mathrm{e}}$ siècle n'hésitaient pas à dire que c'étaient eux les véritables maîtres de l'empire) ; mais dans le cas du ministère de la Justice au moins il est parfaitement clair que les fonctionnaires qui travaillaient dans les bureaux étaient souvent des juristes extrêmement compétents. 


\section{Pierre-Étienne Will}

Nous n'avons que des indications circonstancielles sur le succès de librairie de 1'ouvrage et la demande à laquelle il répondait, mais elles sont malgré tout parlantes. Outre le fait que l'ouvrage a été réimprimé jusque dans les années 1880 - donc sur plus d'un siècle -, il nous est dit que lorsque Zeng Hengde a été nommé dans un poste de préfet en province et qu'il a quitté le ministère en emportant les planches d'impression avec lui, la demande (entendons, à la capitale) a encouragé l'un de ses collègues à produire une nouvelle édition mise à jour. Nous connaissons ces détails grâce à la préface donnée à cette nouvelle édition par un certain Cao Yi 曹 沂, datée de 1791. La date à laquelle Zeng Hengde a quitté la capitale n'est pas précisée, mais Cao, qui est lui-même un ancien fonctionnaire du ministère de la Justice, indique que la nouvelle gravure de l'ouvrage a été entreprise par son père et menée à bien par lui-même. Nous n'avons pas les moyens de savoir si la gravure dont parle Cao est celle de l'édition «de poche » (xiuzhen), encore que cela semble probable; dans tous les cas, la simple existence de telles versions en format réduit, qui sont presque toujours des publications à bon marché produites par des éditeurs commerciaux, est une indication on ne peut plus claire du succès d'un texte et de la demande à laquelle il répondait.

\section{2.}

Cette demande est assez facile à cerner, et c'est elle qui détermine la forme même des publications dont il est ici question. J'y ai déjà fait allusion : les auteurs des recueils de lois et de règlements mis en tableaux affirment d'abord viser un public de débutants, de fonctionnaires frais émoulus qui ont encore tout à apprendre des complications et des dangers de la vie administrative, à qui il convient d'offrir des outils pédagogiques leur permettant de se mettre au courant aussi vite que possible afin d'éviter les erreurs et les ennuis de carrière qui risquent d'en découler. En cela d'ailleurs ils ne font que reprendre un thème tout à fait courant dans les manuels généralistes pour fonctionnaires (les guanzhen 官筬) : le métier 
d'administrateur est difficile et plein de chausse-trappe, et l'on ne saurait s'y lancer sans l'aide de gens expérimentés (comme les muyou) et, d'abord, sans une solide préparation livresque. De nombreux auteurs, dans les préfaces de manuels ou ailleurs, se plaignent de l'inexpérience des « rats de bibliothèque " (shusheng 書生) passés par les examens et propulsés sans transition dans des positions de responsabilité, ainsi que de la totale inadéquation de la formation des lettrés aux tâches concrètes d'un fonctionnaire local, voire même se gaussent de la panique qui saisit le pied-tendre se retrouvant à présider le tribunal au milieu des clameurs contradictoires des parties et du va-et-vient des sbires, et prêt à se laisser manipuler par le premier venu qui lui dira ce qu'il doit faire ${ }^{43}$. De même déplore-t-on le mépris dans lequel les lettrés tendent à tenir les ouvrages techniques, par opposition à ceux qui traitent de la «Voie» : finances, art militaire, agriculture, et surtout, droit, alors que « lire le code» devrait au contraire être le premier souci du futur fonctionnaire. Il se trouve en effet que les considérations que je viens d'évoquer sont particulièrement fréquentes de la part des auteurs ou préfaciers d'ouvrages portant sur le droit et la procédure; or, nous l'avons vu, les recueils de tableaux sont pour leur plus grande part consacrés au code pénal et à son double administratif, les chufen zeli.

Mais il n'y a pas que les débutants. Il est clair que la plupart des recueils de tableaux mentionnés dans la première partie de cet essai visaient au moins autant les administrateurs chevronnés soucieux d'efficacité que ces jeunes sans expérience à qui l'on fournissait, dans le même but, des adaptations versifiées et rimées du code faciles à apprendre par coeur et d'un contenu beaucoup plus simple que les tableaux ${ }^{44}$. Les

\footnotetext{
43 J'en ai donné quelques exemples dans «L'apprentissage du métier de fonctionnaire à la fin de la période impériale », in Christine Nguyen Tri et Catherine Despeux (éd.), Education et instruction en Chine, vol. 2, Paris \& Louvain : Éditions Peeters, 2003, p. 7-47.

44 Je ne puis développer ici sur ces «comptines »- le Dulü ge 讀律歌, le Dulü yide ge 讀律一得歌, le Lüli gejue 律例歌訣, le Dulü guanlang 讀律琯朗, etc. (les titres parlent
} 


\section{Pierre-Étienne Will}

tableaux ne servaient pas seulement à s'initier au code et à la réglementation, c'étaient aussi des outils quotidiens de travail pour les administrateurs, car ceux-ci, à moins d'être de véritables érudits en la matière (il y en avait) ${ }^{45}$, risquaient fort de se perdre dans une réglementation toujours plus foisonnante et embrouillée.

Le texte du code (ne parlons même pas des chufen zeli) est complexe, on a du mal à s'y retrouver, et ce ne sont pas seulement les débutants qui le disent. Dans sa préface à la recension des tableaux de Dong Gongzhen et Shen Xintian qu'il fait paraitre en 1830, Huang Luxi raconte que lorsqu'il a montré le manuscrit aux huit collègues basés à Chengdu prêts à le relire et à contribuer financièrement à sa publication, ces messieurs, qui étaient pourtant tous des «savants d'une grande érudition et particulièrement attentifs aux problèmes judiciaires 》 (皆善讀書究心刑名 之學者), lui ont exprimé dans les termes suivants les difficultés du code et par conséquent l'utilité éminente de son manuscrit :

Les volumes et les sections du Code pénal sont innombrables, chaque fois qu'on s'y plonge on ne peut que soupirer en contemplant un tel océan. Mais à l'intérieur les filaments qui le composent sont dispersés, les articles sont isolés les uns des autres; pour un même type d'affaire les circonstances et la sentence applicable sont chaque fois différentes : si l'on ne procède pas à une vérification minutieuse parmi des détails apparemment semblables, un écart infime risque de vous entraîner à une erreur colossale. Or voici que nous est offert le présent ouvrage, où la consultation par tableaux permet de voir les choses aussi clairement que dans la paume de la main, où pour chaque affaire les éléments correspondants [dans le code] apparaissent lumineusement au premier coup d'œil ! Vraiment, l'on peut bien parler d'un miroir précieux pour ceux qui assument des fonctions !

d'eux-mêmes) -, enrichies ou non, suivant les cas, d'un commentaire en prose, et dont certaines étaient d'ailleurs destinées autant au public des justiciables, qu'on souhaitait sensibiliser aux conséquences de leurs actes, qu'aux administrateurs eux-mêmes.

45 À commencer par les anciens muyou juristes qui avaient réussi à entrer dans la carrière. L'exemple classique est Wang Huizu 汪輝租 (1731-1807), sur lequel voir « L'apprentissage du métier de fonctionnaire... », cf. n. 43 ci-dessus, p. 42. 
Réglementation administrative et code pénal mis en tableaux

以爲律例一書, 篇帙浩繁, 讀者每有望洋之歎, 而其間緀析條分。一事而 情罪各異, 非精審於幾微疑似之間, 則毫䔩之差, 謬以千里。今得是書而 縱横求之瞭如指掌。隨事印證一目了然。誠服官者之筫鑑也。

Quant à Xu Hao, le préfet qui a signé la dernière mouture connue de l'ouvrage, il affirme dans sa préface (de 1869) que c'est seulement après avoir eu accès aux tableaux inventés par Shen Xintian qu'il a commencé à comprendre quelque chose au Code pénal, ou plus exactement à son organisation :

Dès ma jeunesse je lisais le code, mais même à un âge avancé j'étais incapable d'en comprendre le détail. C'est seulement après avoir eu entre les mains l'ouvrage de M. [Shen] que $j$ 'en ai à peu près compris les tenants et les aboutissants. Je n'en ai été que plus convaincu que c'était en disposant les cartes et les annales à leur gauche et à leur droite que les Anciens arrivaient à en tirer mutuellement parti.

余少而讀律, 老而不能詳。得先生之書, 然後稍明其緒。益信古人左右圖 史以相輔而行也。

Je vais revenir dans un instant sur ces cartes et ces annales disposées à la gauche et à la droite des inévitables "Anciens». L'important, pour le moment, c'est de souligner cette idée de clarté et d'immédiateté exprimée par Huang Luxi et par bien d'autres au moyen d'expressions comme " évident au premier coup d'œil » (yimu liaoran 目了然), 《clair comme si on regardait un feu 》 (liao ru guanhuo 燎如觀 火), et ainsi de suite. Et déjà, l'on peut noter qu'il ne s'agit pas nécessairement d'une simple formule rhétorique. Comme le souligne le fanli du Mingfa zhizhang, la mise en page a été calculée pour qu'en effet on saisisse tout «d'un seul coup d'œil » (yiwang liaoran 一望瞭然) : on a cherché autant que possible à faire tenir chaque tableau dans une demi-page (i.e, soit le recto soit le verso d'un folio), mais lorsque la matière était trop importante, au lieu d'être étalée sur toute la surface du folio (recto et verso) - en d'autres termes, au lieu de se trouver sur la même planche d'impression - on l'a partagée entre le verso de la page et le 


\section{Pierre-Étienne Will}

recto de la page suivante, le titre dans la ligne supérieure étant réparti de la même façon (以前頁陰面後頁陽面, 共爲一圖, 標目上格). On ne se trouve donc pas dans la situation courante des tushuo, où il faut tourner la page pour lire au verso les explications accompagnant la planche qui se trouve au recto.

Concernant le même souci de saisie visuelle d'un seul tenant, il est difficile de ne pas parler d'un document exceptionnel mentionné dans certaines préfaces et que je croyais perdu, mais dont, au moment de mettre sous presse, j'ai découvert un exemplaire dans la collection Ōki (大木文庫) à l'Université de Tokyo. Il s'agit d'un set composé de deux tableaux imprimés montés en rouleau, larges de $55,5 \mathrm{~cm}$, représentant d'un seul tenant et avec une densité de détail assez spectaculaire, sur une hauteur de $125 \mathrm{~cm}$, l'essentiel des deux plus grosses sections du code pénal, la section criminelle ( $x$ ing 刑) et la section civile ( $h u$ 戸), plus un certain nombre d'éléments généraux. L'ensemble porte le titre Mingxing tushuo 明刑圖說, et nous savons par d'autres sources que son inventeur était un ancien intendant de circuit au Gansu nommé Tieshan 鐵珊, inconnu par ailleurs. Nous savons aussi que ces tableaux ont servi de source à un ouvrage qui, lui, est assez répandu, le Xuji 續輯 Mingxing tushuo de Hu Taoxuan 胡 陶軒 (préface de 1881), qui résume fort clairement la matière du code et des sanctions relatives à l'administration de la justice en un seul tableau continu (horizontal), relié sous la forme d'un fascicule de 53 doubles pages.

Quoi qu'il en soit, non seulement il faut que ce soit clair, il faut en outre qu'on n'ait pas à perdre de temps en recherches. Pourquoi ? Là encore, la littérature didactique destinée aux administrateurs offre une pléthore de textes et de préfaces insistant sur la nécessité de faire vite, mais sans se tromper. Le risque est toujours grand, dans la fièvre des audiences, alors que les affaires se succèdent et que les plaignants crient justice ${ }^{46}$,

${ }^{46}$ Cf. la préface originale (1740) du Mingfa zhizhang, qui parle de la multitude d'affaires à régler par le magistrat : «Le fonctionnaire en titre doit régler des affaires à longueur d'audience. Dès lors il est nécessaire que les responsables de son cabinet 


\section{Réglementation administrative et code pénal mis en tableaux}

d'édicter dans la hâte un jugement qui sera attaqué en appel (kong 控) par la partie perdante ou rejeté (bo 駁) pour vice de forme ou argumentation insuffisante par les instances supérieures. (Celles-ci sont systématiquement saisies, jusqu'au ministère de la Justice à la capitale pour les crimes impliquant la peine de mort, dès lors qu'il ne s'agit pas simplement de conflits que nous appellerions «civils ».) À cela s'ajoutent bien sûr les sanctions administratives qui frappent toute erreur de jugement et tout manquement à la procédure.

Cela étant, la rapidité n'est pas seulement une contrainte, c'est aussi une vertu, comme le soulignent maints auteurs de manuels, dont certains semblent d'ailleurs avoir été de véritables vedettes des tribunaux. Laisser traîner une affaire ne peut que susciter des problèmes: les pièces à conviction disparaissent, les blessures ou les cadavres à examiner s'altèrent, les parties et les témoins changent d'avis ou se livrent à diverses tractations, les commis et les agents de yamen cherchent bien sûr à s'immiscer dans le processus et à gagner de l'argent sur le dos des justiciables, etc. Simplement, la rapidité exige aussi certaines précautions, dont la première est d'être absolument sûr des bases légales des décisions que l'on prend. C'est à cela que servent les outils de travail qui permettent de débrouiller « au premier coup d'œil » la matière tellement complexe du code et des précédents, quitte à pousser plus avant les recherches lorsque la situation à clarifier devient par trop compliquée sur le plan juridique ${ }^{47}$.

humectent leur pinceau et soupèsent [les circonstances des cas] de l'aube à la nuit » (服 宣者環庭剖決。於斯須司幕者濡筆權衡於旦夜).

${ }^{47}$ Plusieurs auteurs rappellent que les tableaux qu'ils proposent ne contiennent pas la totalité du code et de ses commentaires, et qu'il ne faut pas hésiter à se reporter aux textes complets, ne serait-ce que pour les citer correctement dans les décisions judiciaires. Ainsi le premier article du fanli du Mingfa zhizhang zengding (1743): «Pour les tableaux et explications nous avons adopté une expression simplifiée, afin qu'au moment d'adjuger un cas il soit facile d'invoquer [les provisions du code]; il existe bien sûr un texte complet du Code, et c'est pourquoi nous nous sommes dispensé de citations in extenso " (圖說原取簡便。定案時易于弓証。若律例白有全書。故不連覑繁引). Mieux, le fanli du Mingfa zhizhang xinli zengding compilé par Niu Dawei en 1824 affirme que 


\section{Pierre-Étienne Will}

«Tu»et «Biao »

Cette présentation "claire comme si on l'avait en paume ", donc, prend la forme de «tableaux ». Que faut-il entendre exactement par là ? Remarquons déjà que ce que je m'apprête à décrire plus précisément ne correspond qu'à une toute petite partie du champ sémantique, à vrai dire très vaste, du mot $t u$, lequel désigne aussi bien des figures (des représentations picturales) que des cartes géographiques, des diagrammes, de l'information mise en tableaux (comme dans le cas présent), ou même simplement des listes ${ }^{48}$. Presque tous les ouvrages mentionnés dans la première partie de cet article ont le mot $t u$ dans leur titre ; ou s'ils ne l'ont pas, les tableaux dont ils sont composés sont appelés $t u$ dans la table des matières, ou dans le titre qui surmonte chacun d'entre eux.

Il y a cependant une exception : le dernier ouvrage que j'ai décrit, le Lübiao. Y aurait-il donc une différence entre tu et biao ? Pas à première vue si l'on considère l'ouvrage en question, dont les tableaux se présentent exactement de la même façon que ceux des ouvrages utilisant le mot $t u$. Mais la question mérite néanmoins d'être posée, car l'opposition entre $t u$ et biao fait l'objet de considérations intéressantes de la part de Xu Hao, l'auteur de la dernière recension connue des tableaux de Dong Gongzhen et Shen Xintian, publiée comme nous l'avons vu en 1870 . Cette recension porte le titre Mingfa zhizhang $t u$, mais Xu Hao affirme qu'en fait ce ne sont pas des $t u$, mais des biao:

l'ouvrage ne fait que donner des pistes (yinxian 弓線) et invite avec insistance le lecteur à « ne pas se contenter des tableaux pour prendre des décisions, ce qui pourrait donner lieu à des omissions et à des erreurs » (勿據此 以䨌定, 致有漏, 致有多也).

${ }^{48}$ La mise en regard du Lüli tushuo de Wan Weihan ou du Xingming tushuo de Tiebao, dont il a été question plus haut, et des ouvrages aux titres très proches (Xinglü tushuo 刑 律圖說, Zuiming tushuo 罪名圖說) décrits par Jérôme Bourgon, dans lesquel les tu sont des représentations figurées des peines prévues par le code, illustre parfaitement ce flottement sémantique. Cf. J. Bourgon, «Chinese executions: visualizing their differences with European supplices ", European Journal of East Asian Studies, II/1 (2003), p. 151-182. 
Les Anciens disposaient les cartes à gauche et le texte des annales à droite. Tout ce qui était difficile à décrire dans le texte, ils le dessinaient sous forme de cartes $(t u)$; et les données qui ne pouvaient être entièrement représentées sous forme de cartes, ils les alignaient sous forme de tableaux (biao). Les cartes servent d'appoint pour ce qu'il n'est pas possible de rendre clairement dans un rapport écrit; les tableaux permettent d'éviter que des données complexes n'apparaissent dans le désordre. C'est pourquoi rien ne vaut une carte pour s'enquérir de ce qui est obscur et mystérieux; et rien ne vaut un tableau pour rester simple et éviter la confusion. Les cartes viennent à l'appui du texte des annales ; les tableaux renforcent les cartes. Lorsqu'ils rédigeaient leurs histoires, Sima Qian et Ban Gu attachaient la plus grande importance aux tableaux généalogiques; mais à partir de Chen Shou et Fan Ye on n'a plus été capable d'en composer. ${ }^{49}$

古人左圖右史。凡史所難名狀者, 繪之圖。而圖有不能盡者, 列之表。圖 者所以助擬議之不及也, 表者所以治錯綜之雜出也。是故探蹟索隱莫如圖, 持簡御繁莫如表。圖以佐史, 而表以輔圖。馬班作史並重世表, 陳范以下 即不能票之矣。

Xu Hao place donc les $t u$ et les biao dans deux compartiments bien distincts - impliquant, me semble-t-il, que les $t u$ sont une représentation non textuelle (même si elle peut comporter de la «lettre », comme toute carte géographique), alors que les biao sont simplement du texte réorganisé dans un format particulier. J'aurais plutôt tendance à considérer les biao (tels qu'ils sont décrits ci-dessous) comme un sous-ensemble des $t u$, terme qui, comme on l'a vu, recouvre toutes sortes de formats et de moyens de représentation ; c'est en tout cas ce que semblent aussi avoir pensé la plupart des compilateurs des ouvrages dont nous parlons ici, qui utilisent le terme générique $t u$ pour désigner ce qui, d'après Xu Hao, devrait en fait être appelé biao :

$49 \mathrm{Xu}$ Hao, Mingfa zhizhang $t u$, préface (1869). J'ai hésité entre «cartes» et " planches » pour traduire $t u$, mais dans le contexte de l'écriture de l'histoire, "cartes » me paraît plus satisfaisant. Sima Qian et Ban Gu sont bien sûr les auteurs du Shiji et du Hanshu, Fan Ye et Chen Shou ceux du Hou Han shu et du Sanguo zhi. 


\section{Pierre-Étienne Will}

Sous le règne de Qianlong, $M$. Shen Jiasou [Xintian] de Wuxing composa les «tableaux" (tu) du Mingfa zhizhang ${ }^{50}$ : une ligne horizontale s'étendait au sommet [des tableaux], dont les données étaient arrangées et connectées. Or, un tel format est en réalité un biao.

乾隆中, 吳興沈稼舅先生撰名法指掌圖。旁行变上，排比系屬。按其體例 實表也。

Et Xu Hao poursuit en introduisant la comparaison classique entre l'organisation du code et la structure d'un filet (wang 網), la corde principale (gang 綱) portant la succession ordonnée des lois (liu), et les lois additionnelles ou précédents étant pour leur part comparés aux mailles ( $m u$ 目) qui se raccrochent à la corde. Et de fait, on admettra avec un peu d'imagination que la comparaison entre le filet de la loi et le tableau (biao) n'est pas seulement fonctionnelle, elle est également visuelle: l'entrecroisement des lignes et des colonnes, la ligne de titre en caractères gras qui les surmonte en haut de page, ne peuvent-ils en effet évoquer les mailles et la corde maîtresse du filet ?

Nous verrons dans un instant que dans la plupart des cas les tableaux auxquels nous nous intéressons ici $-\mathrm{y}$ compris ceux publiés par $\mathrm{Xu}$ Hao - sont nettement plus compliqués dans leur organisation que les tableaux appelés biao, formés de lignes et de colonnes régulières, que l'on trouve en effet dans les deux premières histoires dynastiques, ainsi que dans divers autres genres historiographiques : que l'on pense aux tableaux de l'évolution de la structure administrative territoriale (yange 沿革) dans les monographies locales (fangzhi 方志) et les géographies générales ( $y i$ tong $z h i$ 一統志), aux tableaux chronologiques des titulaires des différents postes administratifs d'une localité donnée ou à ceux des lauréats aux examens, également dans les monographies locales, ou encore aux immenses tableaux généalogiques des branches principales et secondaires des ligna-

\footnotetext{
50 À moins qu'il faille comprendre «composa le Mingfa zhizhang $t u$ »; mais l'ouvrage original de Shen Xintian ne comporte pas le mot $t u$ dans son titre.
} 


\section{Réglementation administrative et code pénal mis en tableaux}

ges dans ce qu'on appelle justement, de façon un peu réductrice parce qu'on y trouve beaucoup d'autres choses, les « généalogies 》 (zupu 族譜, litt. « registres claniques »).

Mais qu'il s'agisse de $t u$ ou de biao, cette façon de mettre en tableaux un contenu complexe, ou répétitif, ou les deux à la fois, obéit dans tous les cas à un certain nombre de contraintes et présente certaines caractéristiques communes. Le but recherché est toujours de présenter un ensemble en principe cohérent de données de façon plus lisible et plus pratique, parce que mises en ordre et condensées, que si elles étaient livrées sous forme de texte continu, autrement dit, linéaire: la définition même du tableau, c'est qu'il est à deux dimensions (zongheng chengtu 縱 橫成圖, lit-on parfois), et donc, en principe, à double entrée. Dans un texte « normal », par contraste, il n'y a qu'une seule entrée possible - le début ; ou plus exactement, il n'y a qu'une seule direction possible (à tout le moins en mode d'utilisation normal), à savoir la progression linéaire à partir du point de départ, même si l'on choisit ce point de départ en cours de route.

Au reste, la mise en tableau n'est pas la seule façon concevable d'introduire un minimum de clarté et de hiérarchie dans un texte linéaire. Un tel texte peut être rendu plus facile à consulter et à utiliser en recourant à divers procédés typographiques - ou plus simplement, graphiques, car ils ne sont pas dépendants des techniques d'imprimerie typographique - pour indiquer des départs secondaires, des titres, des hiérarchies, des parallélismes, ou d'autres articulations importantes. Ainsi en va-t-il des retours à la ligne (des paragraphes), des titres mis en évidence par leur positionnement dans le texte ou par leur corps, des changements de corps, ou encore du recours à diverses sortes de ponctuations et de soulignements.

Tout cela existe dans l'édition chinoise traditionnelle, bien sûr. Mais ce qui est intéressant, c'est que dans les ouvrages chinois en impression traditionnelle ces artifices fort utiles - et particulièrement bienvenus pour nous autres lecteurs non chinois - sont le plus souvent réservés aux publications, justement, utilitaires, visant un public peu distingué, et souvent, commerciales : l'idéal d'une belle édition et d'une belle mise en page 


\section{Pierre-Étienne Will}

reste le texte présentant une parfaite continuité à l'œil, remplissant la page de colonne en colonne et sans à-coup, sans ponctuation aucune et sans retour à la ligne ${ }^{51}$. Les articulations logiques et syntaxiques, les changements de partie, etc., c'est au lecteur de les dégager lui-même du texte, de sa forme et de son rythme.

Cette mise en page idéale, qui refuse le moindre artifice de présentation propre à rendre le texte plus facilement accessible, ne se rencontre pas seulement dans l'édition : c'est aussi celle qu'on trouve dans les documents administratifs, à commencer par les plus nobles et les plus formels d'entre eux, les mémoires au trône. Or, elle apparaît particulièrement irrationnelle, voire irritante, lorsque le texte en question est purement énumératif, et tout spécialement lorsque son contenu n'est guère plus que l'équivalent d'un tableau de chiffres. Pour m'en tenir au cas des mémoires au trône, les empereurs Qing, comme on sait, recevaient chaque année des provinces des mémoires rendant compte des chiffres courants de la population et des réserves de grains (les minshu gushu zouzhe 民數穀數奏摺), sur lesquels ils étaient priés d'apposer leur rescrit. Or, dans beaucoup de cas ces mémoires présentaient une comptabilité passablement compliquée, répondant à la logique dite des « quatre colonnes » (sizhu 四柱) - ancienne encaisse, dépenses, recettes, nouveau bilan --, qui appelle presque par définition une présentation en tableau, à tout le moins un retour à la ligne pour distinguer chaque nouvelle rubrique. Mais c'est au lecteur de dégager la structure comptable à partir d'un texte où les seules solutions de continuité sont les retours à la ligne avec élévation chaque fois qu'il est fait allusion au souverain qui en est le destinataire direct ${ }^{52}$.

Ceci n'est qu'un exemple extrême, mais qui trouve beaucoup d'équivalents dans les ouvrages imprimés, à commencer par les publica-

\footnotetext{
${ }^{51}$ Je ne parle pas ici des retours à la ligne avec élévation dans les textes publics faisant allusion à l'empereur et à la dynastie régnante.

52 J'ai proposé une analyse formelle détaillée de ces mémoires dans P.-E. Will et R. Bin Wong, Nourish the People: The State Civilian Granary System in China, 1650-1850, Ann Arbor : University of Michigan Center for Chinese Studies, 1991, chap. 8.
} 


\section{Réglementation administrative et code pénal mis en tableaux}

tions officielles. Dans le cas des guides pour administrateurs, en revanche, ceux surtout qui distillent une information pratique et concrète (par opposition aux ouvrages plutôt axés sur les problèmes d'éthique et d'idéologie), on rencontre fréquemment des modes de présentation, de ponctuation et de mise en page qu'on pourrait dire «utilitaires "; cela est particulièrement vrai des publications dont l'origine est clairement commerciale, qui ne manifestent pas de prétention particulière à la «distinction » sur le plan de la présentation ${ }^{53}$.

\section{«Tu » et « Shuo »}

La mise en tableaux est en quelque sorte le degré ultime de cet effort pour présenter un contenu de façon aussi pratique et aisément utilisable que possible; cela vaut encore plus pour les ouvrages auxquels nous nous intéressons ici, lesquels ne comportent que des tableaux - si l'on excepte bien sûr les préfaces, ainsi que les tables des matières et autres « instructions au lecteur » (fanli). Le plus souvent le corps de l'ouvrage ne comporte pas de «texte» en dehors de celui qui est inclus dans les tableaux eux-mêmes, et qui en forme la matière ; ce texte lui-même n'est pas dans un format « textuel » discursif : c'est de la matière résumée, abrégée au maximum, en style télégraphique pourrait-on dire ${ }^{54}$, et tirant sa signification non de la syntaxe ou de la rhétorique, mais de la disposition spatiale des fragments de texte à l'intérieur du tableau. Nous sommes donc

\footnotetext{
${ }^{53}$ Ce qui n'exclut pas nécessairement que cette présentation soit matériellement soignée. Les productions les plus remarquables de ce point de vue pourraient bien être certaines des éditions du Xiyuan lu jizheng (initialement compilé par Wang Youhuai, comme je l'ai signalé plus haut) parues pendant toute la durée $\mathrm{du} \mathrm{XIX}^{\mathrm{e}}$ siècle : séparation claire des paragraphes, zones de la page réservées à certains types de commentaires, ponctuation abondante et différenciée, utilisation d'encres de plusieurs couleurs pour distinguer les différentes strates de commentaires...

54 À cet égard il faut reconnaître que le style particulier du code pénal, concis à l'extrême et déjà organisé par articles et sous-articles, se prête particulièrement bien à ce type de présentation.
} 


\section{Pierre-Étienne Will}

loin de la formule classique dans laquelle une «illustration » (de quelque nature qu'elle soit) vient à l'appui d'un texte discursif pour le compléter ou l'éclairer. Nous n'avons pas non plus affaire à la combinaison inverse, typiquement dénommée tushuo, dans laquelle un texte vient commenter l'illustration qui le précède (l'image et le texte figurant typiquement au recto et au verso du folio à la chinoise) ${ }^{55}$. Et dans le seul de nos ouvrages comportant effectivement les mots tushuo dans son titre - le Lüli tushuo de Wan Weihan -, il n'y a en fait pas de shuo au sens d' "explication », seulement des $t u \ldots$

Le shuo - le texte -, si l'on veut conserver le mot, devient donc la matière même du tableau. $\mathrm{Du}$ coup, cela implique une transformation du texte d'origine (celui du code - si concis soit-il -, ou des règlements, ou tout autre), non seulement dans sa mise en page et sa typographie, mais souvent aussi dans son organisation et sa logique même, et dans la façon dont on n'en retient que certains fragments. C'est ce que je vais essayer d'illustrer dans le reste de cette section.

\section{Exemples}

Comme on l'a vu, l'objet commun de tous les types de $t u$ ou de biao dont il est ici question est de présenter le plus possible de matière sous une forme physiquement compacte, et en même temps claire et facile à consulter rapidement ${ }^{56}$. Mais on observe des variations considérables suivant les

\footnotetext{
${ }^{55}$ La principale exception est le premier ouvrage que j'ai cité, le Qiangu xingming bianlan de Dong Gongzhen, où les tableaux sont le plus souvent suivi de citations non abrégées de précédents, voire de «cas homologués» (cheng'an 成案) - en ce sens, on peut dire que c'est le « commentaire » qui illustre le tableau, plutôt que l'inverse ; ce type de présentation disparaît pratiquement par la suite.

56 Soulignons au passage que la rapidité de consultation ne tient pas seulement au format même du tableau, elle découle aussi de la logique des regroupements par thèmes, de la précision des tables des matières, des renvois (lorsqu'il y en a), et même des titres inscrits dans les marges centrales, qui permettent de trouver facilement ce qu'on cherche en feuilletant le volume.
} 
ouvrages et en fonction des contenus : il existe en réalité une infinité de formules et de combinaisons, dont je ne puis donner ici qu'un petit nombre d'exemples. Je les ai choisis dans le domaine de la gestion du personnel administratif, où interviennent aussi bien les lois principales et additionnelles du Code pénal (certains comportements dans le cadre des fonctions sont catégorisés comme des crimes) que la réglementation des sanctions administratives.

La formule la plus simple - entendons, celle où la mise en tableau a le moins d'impact sur la forme du texte originel -, est peut-être celle du Lübiao. Zeng Hengde, le compilateur, explique dans le fanli que son but a été de rendre le contenu du Code pénal immédiatement clair en organisant les paragraphes et en les séparant soigneusement par des lignes, et aussi en faisant ressortir l'importance relative des différents contenus par des corps de caractères plus ou moins gros. Dans un tel cas, donc, la mise en tableau n'implique pas d'intervention particulière sur le texte lui-même. C'est, pourrait-on dire, le degré zéro de la réorganisation du matériau d'origine.

On a un degré plus élevé de réorganisation textuelle, sans pour autant que l'opération soit particulièrement complexe car il s'agit d'un contenu extrêmement simple, lorsqu'il s'agit de mettre sous forme de tableau des chiffres, des tarifs ou des barèmes qui sont présentés en texte continu dans les règlements originaux, et peuvent d'ailleurs être dispersés entre plusieurs articles, ou entre des articles promulgués à des dates différentes. On pourrait en donner des quantités d'exemples. La figure 1 reproduit le début d'un tableau du Lüli tushuo de Wan Weihan (section "fonctionnaires », p. 13a) où est donné le type d'information le plus simple qui se puisse concevoir : les distances en $l i$ entre les yamen des principaux fonctionnaires civils et militaires des différentes provinces et la capitale, et, dans le registre inférieur, les délais autorisés (dingxian 定限) pour la transmission des documents ${ }^{57}$. L'information procède colonne après

57 Il s'agit ici de la procédure dans le cadre des enquêtes ordonnées par les ministères en réponse à des mémoires d'accusation (canzou 參奏) pour retard d'un fonctionnaire dans la prise de ses fonctions (cf. plus bas). Le titre du tableau, indiqué dans la marge centrale 
colonne, chaque colonne donnant le nom et la localisation du yamen, la distance, et le délai. On notera les trois lignes horizontales («jusqu'à la capitale ", "[distance en] li », «délai autorisé »), qui permettent d'économiser la répétition des mêmes mots colonne après colonne. Malgré cette astuce de mise en page, on a en réalité affaire à une simple liste, mise en annexe à une présentation plus compliquée de règlements, comme l'indiquent d'ailleurs les mots liehou 列後, «liste ci-après ", à la fin de la dernière colonne de la page précédente. Cette liste cependant présente une particularité par rapport aux textes réglementaires plus formels : elle suit l'ordre croissant des distances (et donc des délais autorisés), et non pas la hiérarchie immuable des provinces que l'on retrouve dans les Institutions des Qing ( $\mathrm{Da}$ Qing huidian 大清會典), dans les géographies générales de l'empire (Da Qing yitong zhi 大淸一統志), etc. ${ }^{58}$

La structure est tout aussi simple dans la figure 2, extraite de la même section du Lüli tushuo (p. 8a), où l'on voit les trois premières lignes d'un tableau consacré aux délais autorisés de voyage pour les fonctionnaires nouvellement nommés (depuis chez eux jusqu'à leur poste) ou promus (depuis leur ancien poste jusqu'au nouveau) ${ }^{59}$. Il s'agit simplement de

du folio 13, est Ziwen daojing chengtu xianqi 咨文到京程途限期 (distances et délais pour l'envoi de communications à la capitale).

58 Sous les Qing cette hiérarchie part bien sûr du Zhili (la province métropolitaine), suivi du terroir ancestral de la dynastie en Mandchourie (Shenging 盛京), du Jiangnan, des provinces de Chine du Nord, du Sud-Est, du moyen-Yangzi et du Sichuan, du Sud, et du Sud-Ouest. Dans la figure 1, l'ordre croissant des distances depuis Pékin fait qu'on passe de la capitale du Zhili (Baoding, à $180 \mathrm{~km}$ au sud), à des yamen situés aux périphéries septentrionales de la même province, au Shandong, au Shanxi, au Henan, etc., non nécessairement regroupés par provinces, et de même dans les pages suivantes, jusqu'aux yamen les plus reculés du Yunnan.

59 "Délais autorisés pour regagner son poste dans le cas des fonctionnaires résidant chez eux en attente d'être nommés ou ayant été promus depuis un poste en province " (Zaiji houxuan ji zaiwai tuisheng furen qixian 在籍候選及在外推浾赴任限期). Le titre en marge est un peu différent : «Délais s'appliquant aux fonctionnaires recevant chez eux 
donner le nombre de jours autorisés entre chacune des dix-neuf capitales provinciales et les dix-huit autres ${ }^{60}$ : ainsi le résident du Zhejiang envoyé au Shanxi ou le fonctionnaire en poste au Henan promu à la capitale sauront-ils à quel temps de voyage ils ont droit à compter du jour où ils ont été notifiés ${ }^{61}$, au-delà duquel ils encourront retenues de salaire et autres désagréments. Chaque ligne du tableau indique par conséquent, dans le registre supérieur, le nom de la province considérée suivi du mot « jusqu'à », dans le registre médian la liste des autres provinces, et dans le registre inférieur le nombre de jours de voyage autorisés vers chaque province. L'ordre des provinces dans le registre médian ne semble avoir pour but que de regrouper celles pour lesquelles ce nombre est le même, et donc de n'avoir à le faire apparaitre qu'une seule fois ${ }^{62}$.

Dans de tels exemples on a une correspondance univoque entre un lieu (qui est le mode d'entrée unique du tableau) et un ou plusieurs chiffres. Mais la plupart du temps les choses sont beaucoup plus compliquées. Il n'est que de consulter, toujours dans le Lüli tushuo, le tableau qui suit immédiatement celui dont il vient d'être question, et qui est consacré, lui, aux sanctions applicables à quiconque «n'a pas respecté les délais » (weixian 違限) indiqués dans le tableau précédent. On aperçoit dans la première page de ce tableau (figure 3) la façon dont l'auteur s'efforce de faire apparaître tous les cas de figure concevables. Le haut de chaque colonne indique la catégorie de retard: (1) fonctionnaire ayant signé son

leur nomination et à ceux qui sont promus depuis un poste en province » (在籍領 憑在外 推郬限期).

${ }^{60}$ Il y a une petite irrégularité dans le cas du Zhili, où c'est presque toujours Shuntian (i.e. Pékin) qui est indiqué, plutôt que Baoding, la capitale de la province.

${ }^{61}$ Plus précisément à partir du jour où ils se sont mis en route, car ils ont droit à un délai (d'un mois dans le cas des nouveaux nommés, de dix jours dans le cas des promus) entre le moment où ils sont officiellement notifiés et celui où ils doivent entreprendre leur voyage.

${ }^{62}$ On aurait pu s'attendre à ce que les délais soient toujours alignés par ordre croissant (comme dans la première et la troisième lignes de la figure 1), mais ce n'est pratiquement jamais le cas. 


\section{Pierre-Étienne Will}

document de nomination en retard (huaping weixian 畫溤違限), (2) ayant regagné son poste en retard après avoir reçu son document (lingping furen weixian 領憑赴任違限), (3) cas particulier des fonctionnaires d'éducation (jiaozhi 教職), lesquels reçoivent leur document de nomination directement du trésorier de la province (buzhengsi 布政司) ${ }^{63}$, (4) fonctionnaire ayant été reçu en audience par l'empereur (yinjian guanyuan 引見官員) et regagnant son poste en retard, et enfin (5) fonctionnaire en ayant terminé avec une mission ad hoc (fengchai shijun 奉差事竣) et regagnant son poste en retard. Le détail des sanctions est indiqué plus bas dans chaque colonne.

Dans le cas (2), par exemple, la colonne se divise en deux pour indiquer la sanction en fonction de la durée du retard : si c'est moins d'un mois, on est dispensé de sanction (mianyi 朶議), si c'est six mois, on est cassé de sa charge (gezhi 革職), et dans l'entre-deux on encourt des retenues de salaire (fafeng 罰俸) ou, plus grave, des mutations (diaoyong 調 用) avec diminution de grade (jiangji 降級); la partie inférieure de la colonne contient un petit texte sur les conditions dans lesquelles on a droit à un délai supplémentaire, mais seulement dans certaines limites, si on est tombé malade en route, tout en prévoyant des sanctions pour le fonctionnaire comme pour son garant si le certificat établissant la réalité de la maladie s'avère inexact. Dans le cas des fonctionnaires d'éducation (3), il y a deux sortes de délais, avec sanctions assorties en cas de dépassement: celui dont dispose le trésorier provincial pour remettre sa nomination à l'intéressé une fois qu'il l'a reçue du ministère, et celui dont dispose l'intéressé pour gagner son poste; s'il est en retard, son cas tombe sous le précédent général des fonctionnaires ayant dépassé le délai imparti après avoir reçu leur document de nomination. Il en va de même dans les cas (4) et (5). Enfin, le bas de la colonne (4)-(5) est réservé à une définition générale de la notion de délai imparti après remise d'une nomination (pingxian

\footnotetext{
${ }^{63}$ Le « trésorier provincial » est en fait le chef de l'administration de la province, placé juste en dessous du gouverneur.
} 
憑限) ${ }^{64}$. Ce tableau se poursuit sur deux autres pages où apparaissent d'autres cas de figure et où sont fournies toutes sortes d'indications sur la procédure, les délais de transmission de documents pour les nominations et pour les censures en cas de retard, les sanctions en cas de dépassement des délais dans diverses situations, etc., qu'il est inutile d'exposer ici en détail.

Les choses se compliquent d'une autre manière lorsqu'un tableau combine une liste de chiffres du même type que celles reproduites dans les figures 1 et 2 avec des données qualitatives non chiffrées. C'est ce qu'illustre le tableau de la figure 4, extrait de la section sur les « six biens mal acquis » (liuzang 六呀) du Mingfa zhizhang xinli zengding de Niu Dawei (1824) et portant sur les « détournements par un agent de contrôle ou un agent ayant la garde [des biens détournés] » (jianshou dao 監守盜) - un de ces tableaux sur deux demi-pages se faisant face dont j'ai parlé. On est là dans le domaine non plus des fautes administratives, mais bien des crimes sanctionnés par le code pénal. Le tableau présente deux registres. Le registre supérieur a la forme d'un tableau à une entrée, avec dans la première ligne les chiffres de biens détournés (en onces d'argent), et dans la seconde les peines correspondantes. Les mêmes données se trouvent en texte continu - chaque chiffre suivi de la peine correspondante - dans l'article du Code pénal intitulé (dans la traduction du P. Boulais) «Inspecteurs et gardiens volant l'argent et le riz des greniers et des trésors publics 》 (監守自盜倉庫錢糧) ${ }^{65}$. La première case de la première ligne reproduit (en inversant l'ordre original) deux phrases du début de l'article,

${ }^{64}$ De même le bas de la colonne (1) donne-t-il quelques indications générales sur la remise du document de nomination (jiaoping 繳諰) aux autorités provinciales lorsque le fonctionnaire arrive au terme de son voyage, ainsi que sur le problème des documents de nomination souillés, endommagés ou perdus.

${ }^{65}$ Guy Boulais, Manuel $d u$ Code chinois, Shanghai: Imprimerie de la mission catholique, 1924, p. 477-478. Voir aussi Xue Yunsheng 薛允升, Duli cunyi 讀例存疑 (Doutes persistants à la lecture des lois additionnelles, 1905), Taipei : Chinese Materials Center, 1970, vol. 3, p. 577 sq. (loi 264/00). Il s'agit d'un article inclus dans la section zeidao 睋盜 (《brigands et voleurs ») de la partie «peines» (xingbu 刑部) du Code pénal. 


\section{Pierre-Étienne Will}

«la peine [pour chaque individu] est calculée en fonction du total des détournements [s'il y a plusieurs personnes impliquées] », et « il n'est pas fait de distinction entre les meneurs et les comparses $»$. La première case de la deuxième ligne précise que les fonctionnaires se voient épargner le tatouage des caractères " voleur de biens publics » sur l'avant-bras, également prévu par l'article du code - détail important, puisque après tout il s'agit d'un manuel pour fonctionnaires.

Le registre inférieur, quant à lui, est une suite de définitions s'appliquant à ce tableau de chiffres, à savoir les circonstances extrêmement nombreuses dans lesquelles on est passible d'être condamné parce $q u$ 'on a commis des actes correspondant à la définition de l'article du code en question (raisonnement en $y i$ 以), et celles où l'on est passible d'être condamné au même titre que si on avait commis de tels actes (raisonnement en zhun 准) ${ }^{66}$. Ces définitions, qu'on ne trouve pas dans les commentaires du code, sont probablement extraites de " cas homologués » (cheng'an 成案). Les données concentrées dans un tel tableau sont donc d'origines diverses et, pour la partie inférieure, relativement complexes. Ajoutons que, pour l'utilisateur de l'ouvrage, c'est certainement cette partie inférieure qui était du plus grand intérêt dans la mesure où elle regroupe une quantité d'informations difficiles à trouver, car dispersées dans de vastes recueils de cas, alors que les chiffres de la partie supérieure sont donnés dans n'importe quel manuel sur le Code pénal.

La figure 5, extraite du Lüli tushuo zhangzhen (l'ouvrage concurrent de celui de Wan Weihan, dénoncé par ce dernier comme un infâme piratage), donne à voir un autre type d'organisation. Il s'agit des cas d'esclaves cherchant frauduleusement à acquérir des charges mandarinales en échange de contributions, alors qu'en principe leur statut le leur inter-

\footnotetext{
${ }^{66}$ Sur ces modes de raisonnement et les « huit caractères », voir Jérôme Bourgon, «Les vertus juridiques de l'exemple : nature et fonction de la mise en exemple dans le droit de la Chine impériale », Extrême-Orient, Extrême-Occident, 19 (1997), p. 7-41, notamment p. $20 s q$.
} 
dit $^{67}$. Si l'on excepte le bas du tableau, qui traite des jiapu 家僕, c'est-à-dire de serviteurs attachés à une famille mais libres de reprendre leur indépendance, dont il est simplement dit qu'ils n'y a pas lieu de leur appliquer toutes les rigueurs de la loi et qu'ils s'en tireront avec 80 coups de bâton, le reste, qui concerne les esclaves en général, est divisé en deux colonnes qui doivent se lire l'une à la suite de l'autre, donc de façon linéaire. Le haut de la première colonne indique la qualification pénale et la peine : la qualification est «par analogie (bizhao 比照) avec [la loi additionnelle relative à] quiconque étant exclu par la loi des sélections [de fonctionnaires] a camouflé sa situation publique et privée et a changé son nom dans le but d'être sélectionné, mais n'a pas encore été nommé », et la peine est l'exil militaire ${ }^{68}$. Puis viennent les sanctions administratives (renvoi de la charge, retenues de salaire, diminutions de rang) frappant (1) les fonctionnaires qui ont "garanti mutuellement» le candidat frauduleux ${ }^{69}$, à propos desquels est cité un précédent de 1735, (2) les fonctionnaires locaux qui lui ont donné les documents nécessaires pour se rendre à la sélection à la capitale ainsi que les fonctionnaires métropolitains originaires de la même province qui lui ont donné leur garantie, à chaque fois sans se soucier de vérifier son identité réelle, et enfin (3) le

\section{${ }^{67}$ Lüli tushuo zhangzhen, 吏, p. 12, «Nupu juanna »奴僕捐納.}

${ }^{68}$ Cette analogie repose probablement sur des «cas homologués» ou des édits impériaux ayant valeur de précédent. Le texte cité dans le tableau consiste en fragments d'une loi additionnelle beaucoup plus longue, relative aux fonctionnaires et commis qui ont été punis, ou rayés des cadres, et n'ont le droit ni de se représenter ni de racheter une charge, mais ont néanmoins réussi à camoufler leur situation par divers moyens, et se sont fait prendre. Cf. Duli cunyi, vol. 2, p. 194 (loi additionnelle 053/01); Boulais, Manuel du Code chinois, p. 122, qui ne cite le texte qu'en abrégé. On notera l'imprécision de la peine indiquée dans le tableau (" envoyés servir dans une garnison frontalière "), alors que la loi précise que pour ceux «qui n'ont pas été encore nommés » (dont il est ici question) il s'agit d'un « exil militaire très rapproché » (附近充軍), par opposition à l'《 exil militaire rapproché » (近邊充軍) pour ceux qui ont déjà été nommés.

69 Tout candidat en «attente de sélection » (houxuan 候選) devait présenter une garantie signée par cinq fonctionnaires de sa province d'origine. 


\section{Pierre-Étienne Will}

gouverneur général et le gouverneur de sa province d'origine, dont la responsabilité est hiérarchiquement engagée ${ }^{70}$. Là encore, l'intérêt du tableau est de combiner des informations de provenance variée (pénale et administrative) mais concernant un même type de problème.

Le tableau reproduit dans la figure 6 , qui fait suite au précédent dans le même ouvrage, concerne les promotions à des charges subalternes dans les rangs du fonctionnariat auxquelles pouvaient prétendre les commis (liyuan 吏員) méritants avec au moins cinq ans de service - une provision dont on se demande d'ailleurs si elle était souvent appliquée ${ }^{71}$. L'organisation des zones du tableau est un peu plus compliquée, mais elle suit le même principe: les trois colonnes principales, éventuellement subdivisables pour distinguer des cas de figure différents, doivent se lire l'une à la suite de l'autre. La différence par rapport au tableau précédent est qu'on ne part pas d'un crime, ou d'une fraude, mais d'un exposé de la réglementation, les comportements y contrevenant et les sanctions ne venant qu'ensuite. On trouve donc (1) au début, des zones consacrées à la réglementation proprement dite (dans quelles conditions les commis peuvent prétendre à ces avantages, leur classement en quatre catégories à la suite d'un examen par le gouverneur de la province, et les détails de la procédure); (2) puis des zones consacrées aux peines de bastonnade et d'exil sanctionnant ceux qui ont passé les examens spéciaux en recourant à diverses formes de fraude, ou ont menti sur leur identité, ou sur la durée de leur service - on est donc là dans le domaine pénal, et il est fait à chaque fois référence à la loi invoquée par analogie ; (3) enfin, s'intercalant avec les précédentes, des zones consacrées aux sanctions administratives frappant les fonctionnaires qui ont manqué de vigilance.

À titre de comparaison, la figure 7 reproduit la page du « vrai » Lüli tushuo, celui de Wan Weihan, consacrée au même sujet. Comme on le voit,

\footnotetext{
${ }^{70}$ Notons que des sanctions pénales étaient susceptibles de s'appliquer à ces mêmes fonctionnaires en cas de corruption avérée, c'est-à-dire s'ils avaient touché des pots-de-vin pour couvrir la fraude, mais ce n'est pas indiqué dans le tableau.

${ }^{71}$ Lüli tushuo zhangzhen, 吏, p. 13, «Liyuan kaozhi » 吏員考職.
} 
le contenu est exactement le même, mais la disposition des zones est légèrement différente, et en fait la mise en page et la typographie sont moins claires : d'une certaine manière, l'imitation est plus réussie que l'original ${ }^{72}$.

Aucun des tableaux présentés jusqu'ici ne peut être décrit à proprement parler comme un tableau à double entrée. C'est le cas en revanche des deux tableaux présentés dans la figure 8, extraits du Mingfa zhizhang tu de Xu Hao (1870), en d'autres termes la dernière mouture de la série initiée en 1734 décrite plus haut. Il s'agit ici uniquement du Code pénal, en l'occurrence de la section "homicides" (renming 人命) dans la partie «peines » (xingbu). Le tableau de droite concerne encore les fonctionnaires, mais cette fois comme victimes puisque il y est question des "assassinats prémédités de fonctionnaires ou d'envoyés impériaux " (mousha guanshi 謀殺官使). Les lignes correspondent au degré d'aboutissement: tentative sans blessures infligées (已行未傷), tentative avec blessures infligées (已傷), homicide effectif (已殺). Les colonnes correspondent au statut de l'assassin (ou des assassins ${ }^{73}$ ) et de la victime: fonctionnaires locaux ayant prémédité l'assassinat d'un envoyé spécial de l'empereur, administrés ayant prémédité l'assassinat de leur préfet ou magistrat, soldats ayant prémédité l'assassinat de leur officier, commis ou licteurs ayant prémédité l'assassinat d'un fonctionnaire du $5^{\mathrm{e}}$ rang ou au-dessus dont ils dépendent. Les peines prévues par le code sont obtenues en croisant ces deux sortes de critères; ils font en outre la distinction entre instigateurs et comparses (shoucong 首從) et, dans le cas des seconds, entre ceux qui ont activement prêté la main à la tentative d'assassinat (jia-

\footnotetext{
${ }^{72}$ Lüli tushuo, ibid, p. 54a. On notera toutefois, dans le grand cadre exposant la procédure, que l'original (figure 7) ne comporte pas la coquille que l'on trouve dans la copie (每月七月 au lieu de 每年七月).

${ }^{73}$ Un commentaire affirme que dans un homicide prémédité (mousha) il faut qu'il y ait au moins deux personnes (謀殺之因, 必起于二人), d'où la distinction entre l'instigateur (首, ou 造意) et le(s) comparse(s) (從, ou 悉從) dans le texte de la loi. Cf. Shen Zhiqi 沈 之奇, Da Qing lüjizhu 大清律輯註 (1715), Pékin : Falü chubanshe, 1998, vol. 2, p. 659.
} 


\section{Pierre-Étienne Will}

gong 加功), ceux qui n'y ont pas activement prêté la main (bu jiagong), et ceux qui n'ont pas participé à l'acte (buxing 不行), i.e. qui n'étaient pas présents. Comme on peut le voir, la peine maximum - décapitation immédiate $-s$ 'applique aux instigateurs et comparses actifs dans les colonnes 2-4 de la troisième ligne (homicide effectif). Enfin, les deux dernières colonnes du tableau prévoient (1) le cas des commis, soldats ou gens du peuple ayant assassiné des fonctionnaires locaux du $6^{\mathrm{e}}$ rang et en dessous, mais dont ils ne dépendent pas, cas assimilé à la catégorie générale des assassinats prémédités; et (2), le cas des assassinats prémédités de fonctionnaires en poste à la capitale, qui entraîne systématiquement la décapitation immédiate.

Il n'est pas inintéressant de comparer cette mise en tableau au texte original de la loi, qui présente les mêmes données sous forme linéaire et avec des regroupements un peu différents. Je le donne dans la traduction du P. Boulais, bien qu'elle ne soit pas parfaitement littérale :

[Meurtre prémédité d'un envoyé impérial et d'un mandarin]. On infligera les peines suivantes à tout meurtre prémédité commis $1^{\circ}$ par un mandarin ou un employé de son tribunal à l'égard d'un délégué impérial envoyé dans le lieu de sa juridiction ; $2^{\circ}$ par un simple particulier à l'égard de son préfet ou sous-préfet ; $3^{\circ}$ par un soldat à l'égard de l'officier auquel il est soumis ; $4^{\circ}$ par un employé ou un valet de tribunal à l'égard de son propre mandarin, pourvu toutefois que ce mandarin soit du $5^{\mathrm{e}}$ degré au moins :

On condamnera à 100 coups de bâton et à l'exil perpétuel à 2000 lis celui qui aura commencé d'exécuter son projet, sans cependant causer la moindre blessure; on étranglera celui qui aura causé une blessure ; on décapitera celui qui aura accompli son meurtre.

(Les coupables secondaires, qui n'auront ni prêté main forte aux assassins, ni commencé la mise à exécution de leur crime, et ceux qui auront tué avec préméditation un mandarin du $6^{\text {e }}$ degré au plus, un préfet, un sous-préfet ou un officier d'un autre département ou arrondissement, recevront la peine portée contre les homicides ordinaires (strangulat. après les assises, comm. off. ${ }^{74}$.

\footnotetext{
74 Manuel du Code chinois, p. 543-544.
} 
[謀殺制使及本管長官]。凡奉制命出使, 而所在官吏謀殺, 及部民謀殺本 屬知府、知州、知縣, 軍士謀殺本管官, 若吏卒謀殺本部五品以上長官, 已行 未傷者, 首杖一百, 流二千里; 已傷者, 首絞; 流絞俱不言皆, 則舀從各隇等, 官吏 謀殺監候, 餘皆決不待時, 下斬同。已殺者, 皆斬。其從而不加功與不行者, 及謀殺六品 以下長官, 並府州縣任氞首領官, 其非本屬本管本部者, 各依凡人謀殺諭。7

On voit donc que dans le tableau du Mingfa zhizhang $t u^{76}$, le texte a été simplifié par endroits, mais que certains éléments des « notes en petits caractères » (xiaozhu 小註) ${ }^{77}$ ont été explicités (en précisant la peine exacte au lieu de dire simplement «diminution d'un degré ») et redistribués dans chaque case; de même une colonne spéciale (la première) a-t-elle été réservée pour expliciter le fait, sommairement mentionné dans une note en petits caractères, que les fonctionnaires assassins d'un émissaire impérial ont droit à la strangulation ou la décapitation après les assises (jianhou 監候) alors que les subalternes ou les simples particuliers, qui font l'objet des colonnes (2)-(4), sont exécutés sans délai (jue 決) ${ }^{78}$. Le cas des soldats, sbires et simples particuliers qui assassinent un fonctionnaire de rang 6 ou en dessous dont ils ne dépendent pas, mentionné à la fin de la loi dans une note en petits caractères, est placé dans une colonne à part dans le tableau. Enfin, je n'ai pas identifié l'origine de la dernière colonne, relative au meurtre prémédité d'un fonctionnaire exerçant à la capitale.

Des remarques similaires pourraient être faites à propos du tableau de gauche dans la figure 8 , qui concerne les assassinats prémédités des ânés par des membres de la famille (mousha zunzhang 謀殺尊長) et

\footnotetext{
${ }^{75}$ Duli cunyi, vol. 4, p. 789 (article 283/00).

${ }^{76}$ Ou d'autres recensions du même ouvrage, en dépit de quelques variantes dans la disposition graphique de l'information qu'il est inutile de présenter ici.

77 Celles-ci ont été intégrées au texte de la loi - repris des Ming à peu de choses près au début des Qing, mais on continue de les distinguer par la typographie.

${ }^{78}$ Un grand nombre de condamnations à mort "après les assises »-i.e. réexaminées aux Assises d'automne (qiushen 秋番) auxquelles présidait l'empereur - étaient en fin de compte commuées.
} 
correspond à l'article du code venant juste après celui sur les assassinats de fonctionnaires ${ }^{79}$. Ce tableau présente exactement la même structure que le précédent, mais la combinatoire est un peu plus compliquée en raison des distinctions entre les catégories de deuil auxquelles appartiennent les meurtriers par rapport à la victime, du problème des parents par alliance, et du cas particulier des esclaves et des employés commettant des crimes contre les membres de la famille. Le texte original de la loi (plus long que dans le cas précédent, et que je ne reproduis pas ici) a été abrégé et redistribué à l'intérieur du tableau de la même façon. Certains éléments ont été empruntés à des lois additionnelles, comme par exemple le problème des comparses n'appartenant pas à la famille (pangren 旁人) dans l'avant-dernière colonne, ou le degré de participation des comparses au meurtre dans la dernière ${ }^{80}$. On notera enfin que, dans un souci évident de plus grande clarté, la deuxième partie de la loi, qui concerne la situation inverse - les assassinats prémédités de «juniors » par des « seniors »-, a été omise du tableau $^{81}$ : elle est traitée dans un tableau à part, à la suite, intitulé «assassinats prémédités de parents plus jeunes » (mousha beiyou 謀殺卑 幼).

${ }^{79}$ Le titre exact de cette loi est « assassinat prémédité des grands-parents ou des parents 》 (mousha zufumu fumu 謀殺租父母父母): cf. Duli cunyi, vol. 4, p. 780-781 (article 284/00), et Manuel du Code chinois, p. 544 ( Parricide prémédité »). L'assassinat des « seniors » à l'intérieur de la famille était considéré comme moralement plus condamnable encore que l'assassinat d'un fonctionnaire : c'était le quatrième des “dix crimes abominables» (shi'e 十惡), appelé eni 惡逆 (Boulais : “détestable subversion »). C'est ce qui explique que la peine maximale soit ici la plus grave concevable, la mort lente par dépeçage (lingchi 凌遲).

${ }^{80}$ Ces emprunts sont absents du tableau sur les assassinats de fonctionnaires, car la loi concernée n'a pas de lois additionnelles attachées.

${ }^{81}$ La loi est en trois parties : la première porte sur les assassinats des grands-parents ou des parents par des membres plus jeunes de la famille, la troisième est consacrée au cas des esclaves et des employés. 
On pourrait poursuivre longtemps, tant sont variés les contenus et les formules graphiques choisies pour les représenter dans les différentes sortes d'ouvrages que j'ai énumérées. Il me semble néanmoins que les quelques exemples qui précèdent devraient suffire à mettre visuellement en évidence quelques-unes des caractéristiques déjà soulignées : la combinaison de réglementations d'origine hétérogène - le code pénal, la législation sur les sanctions administratives, voire la jurisprudence des « cas homologués » - à l'intérieur d'un même espace soigneusement dessiné et organisé ; l'effort pour mettre graphiquement en évidence tous les cas de figure dans l'enchaînement des causes et des conséquences (des délits et des sanctions), où les choses deviennent vite compliquées, en particulier dans le domaine couvert par les chufen zeli; et enfin, l'ambition de faire tenir dans des ouvrages de format réduit et de consultation facile la plus grande quantité possible de matériaux réglementaires et juridiques.

On peut se demander, pour finir, pourquoi de tels ouvrages n'apparaissent qu'à partir du XVIII siècle, pour continuer de fleurir au siècle suivant. Je n'ai de réponses que très générales. Ni le code pénal avec ses commentaires, ni les précédents (devenus lois additionnelles à partir du code de 1740), ni les sanctions administratives ne sont des inventions des Qing. Mais il est incontestable que le règne de l'empereur Yongzheng (1722-1735), dont les efforts pour reprendre la bureaucratie en main et mettre de l'ordre dans l'administration de l'empire sont bien connus, a marqué le début d'un processus de centralisation et de systématisation accrues de l'appareil réglementaire. Ce processus, qui s'est poursuivi jusqu'à la fin de la dynastie et dont la prolifération des textes est une conséquence directe, ne reflétait pas seulement le projet «légiste » de renforcer le pouvoir dynastique en étendant aux moindres détails le contrôle du pouvoir central sur le travail des fonctionnaires. Il découlait d'abord de la croissance rapide de la population et de l'économie de la Chine : dès les premières décennies du XVIII ${ }^{\mathrm{e}}$ siècle, la masse à administrer est sans commune mesure avec ce qu'avaient connu les régimes précédents, et les problèmes de contrôle et de régulation que cela pose augmentent en proportion. 
On a souvent affirmé que l'effectif de la fonction publique à la fin de l'empire n'a pas suivi cette croissance et que, par conséquent, la Chine s'est trouvée administrée de moins en moins par les représentants directs de l'État et de plus en plus, si l'on peut dire, en sous-traitance, par des instances privées comme les guildes ou les lignages ${ }^{82}$. Je suis convaincu que rien n'est moins vrai. S'il est exact que le nombre des postes «titulaires » dans l'appareil d'État est resté plus ou moins constant, en revanche la force de travail administratif à la disposition des gouvernements provinciaux sous la forme de fonctionnaires en attente de nomination (de "stagiaires ») a cru énormément, particulièrement au XIX ${ }^{\mathrm{e}}$ siècle, et plus encore le nombre de ces professionnels authentiques de l'administration locale qu'étaient les conseillers techniques travaillant dans les cabinets des fonctionnaires en poste et payés par eux, c'est-à-dire en fin de compte par les usagers. C'est grâce à eux que l'État dynastique a pu maintenir et, en fait, accroître ses ambitions en matière de régulation sociale, dans le domaine juridique tout particulièrement. La croissance des règlements et de la jurisprudence, qu'on aurait tort de considérer comme un phénomène déconnecté de la réalité et profitant uniquement à des subalternes malhonnêtes, en est la manifestation la plus immédiate, et la multiplication exponentielle des ouvrages pédagogiques et des outils de travail à l'usage des administrateurs à la même époque va évidemment de pair. Les mises en tableau de la législation ne représentent qu'une fraction limitée de cette littérature. Il m'a néanmoins paru intéressant de les étudier car il s'agit d'un genre découlant directement des développements que je viens d'évoquer, et dont le succès semble avoir été grand, et qu'il n'a fait jusqu'ici l'objet d'aucune attention.

\footnotetext{
${ }^{82}$ La référence classique là-dessus est George William Skinner, «Introduction : Urban Development in Imperial China », in Skinner (éd.), The City in Late Imperial China, Stanford, Stanford University Press, 1977, notamment p. 19-23. Skinner fonde son raisonnement sur le nombre d'administrations sous-préfectorales ( county-level units »), qui est en effet resté à peu près au même niveau pendant toute l'histoire de l'empire, quand il ne décroissait pas.
} 
Réglementation administrative et code pénal mis en tableaux

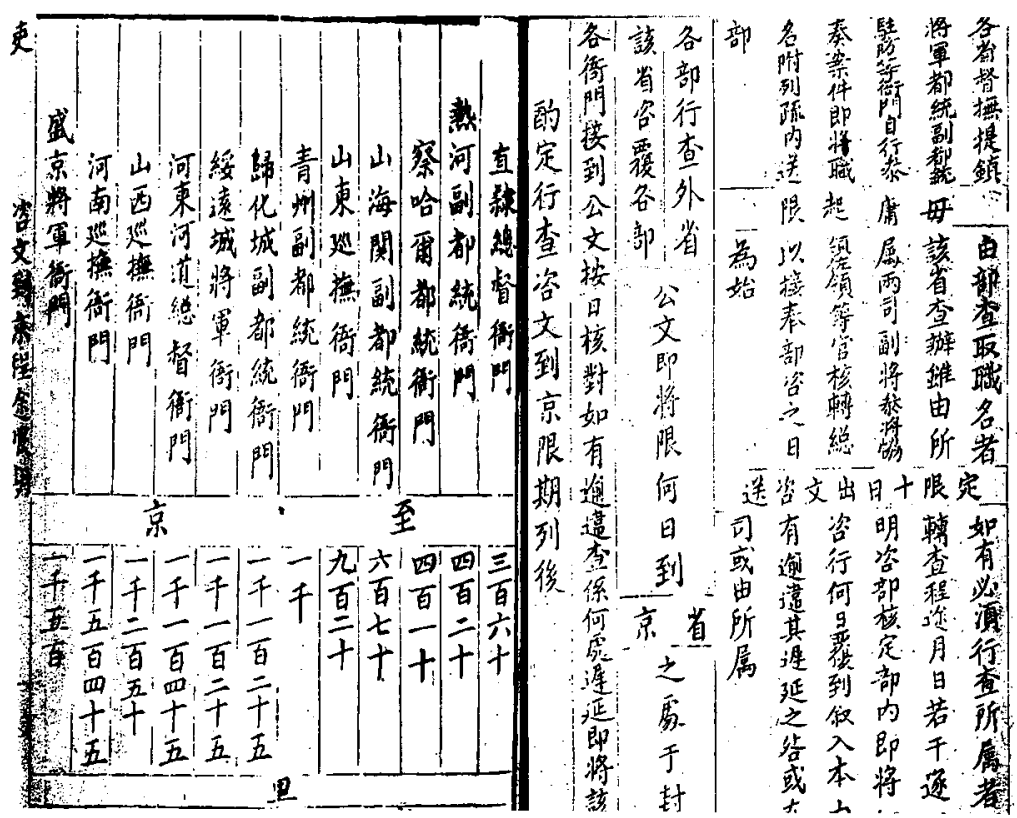

figure 1 


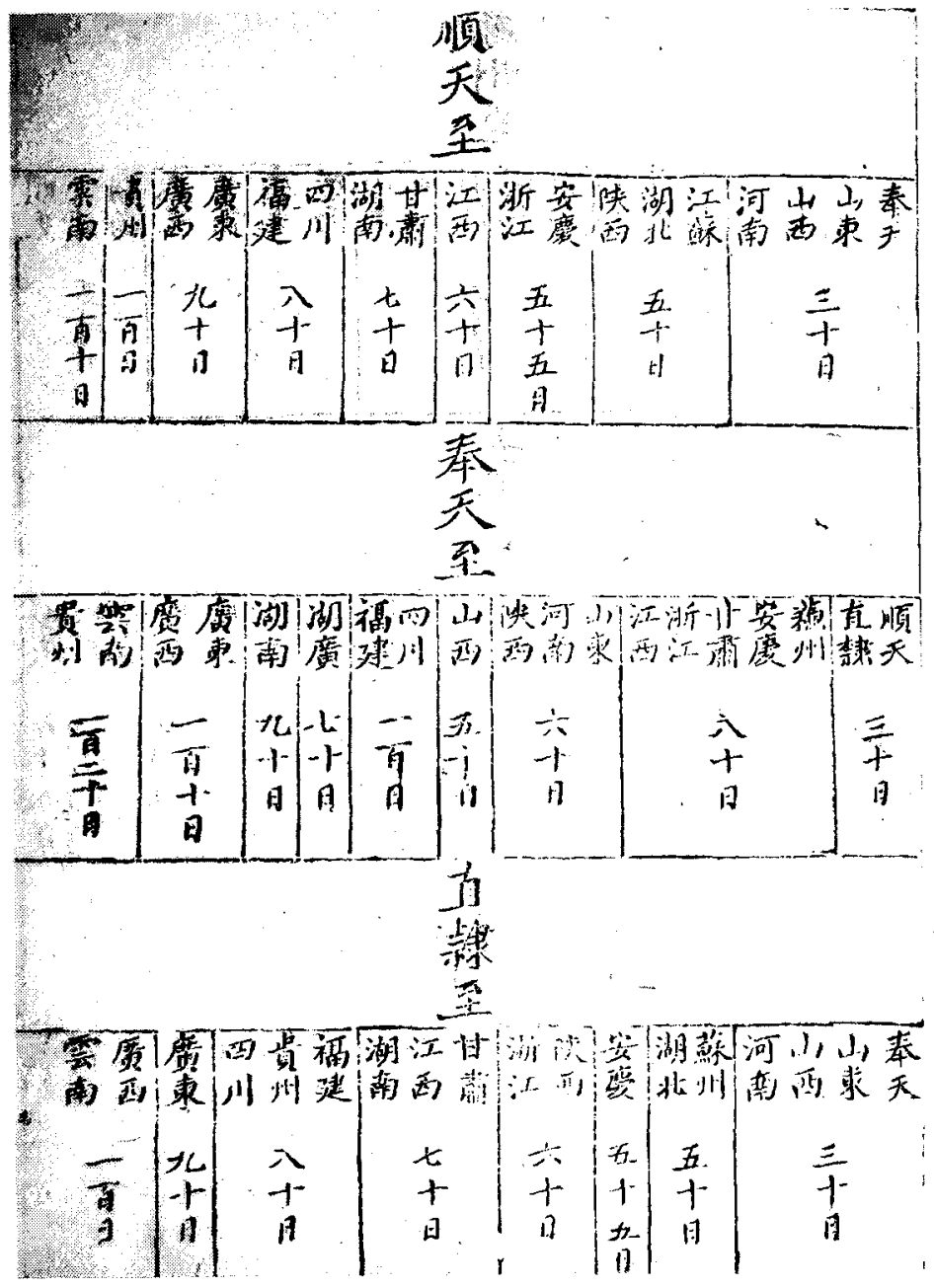

figure 2 
Réglementation administrative et code pénal mis en tableaux

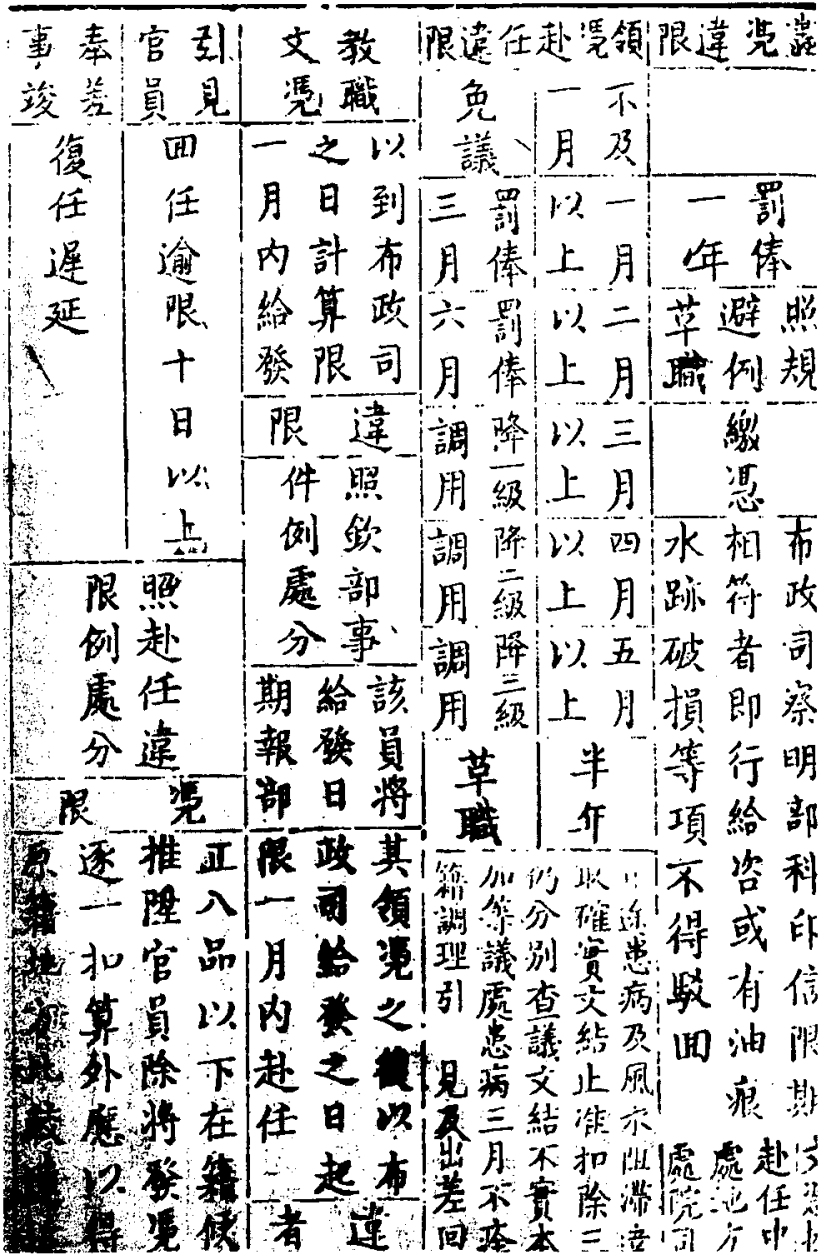

figure 3 


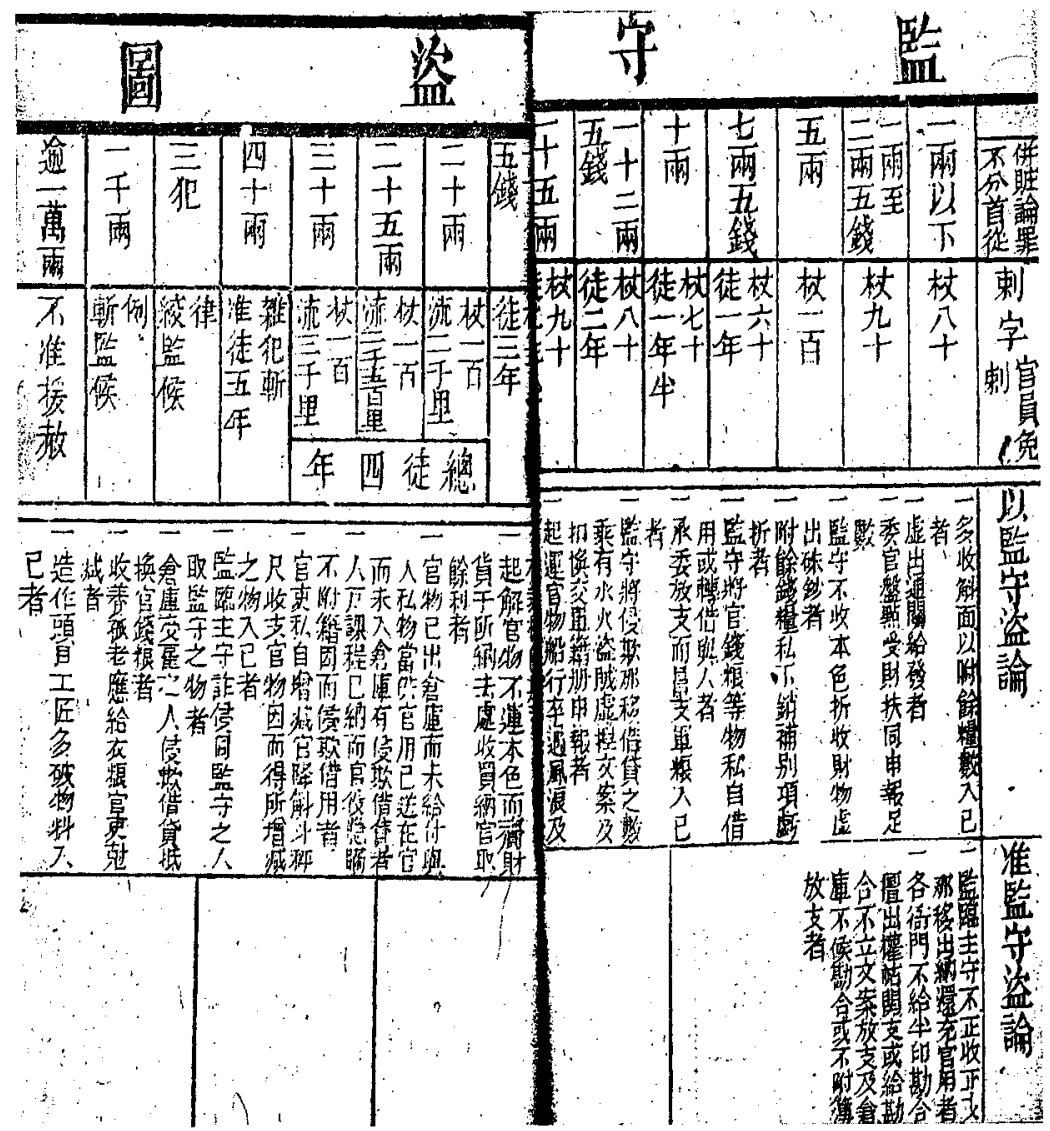

figure 4 
Réglementation administrative et code pénal mis en tableaux

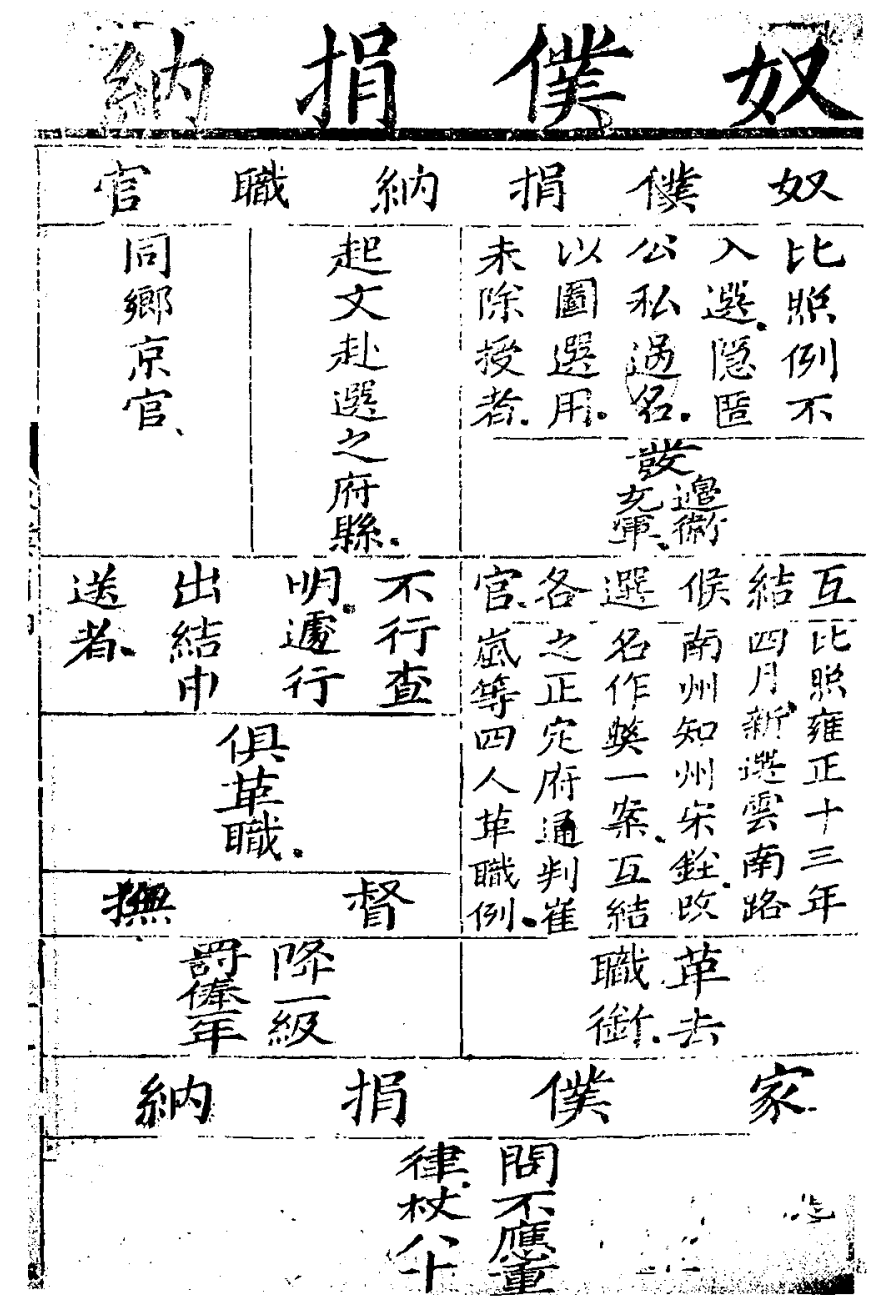

figure 5 

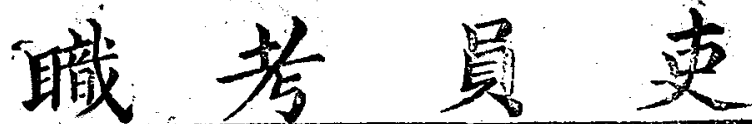

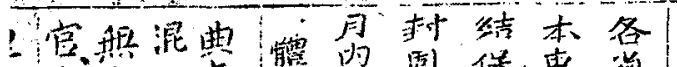

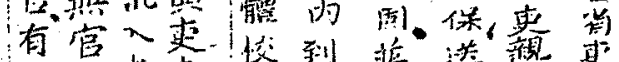

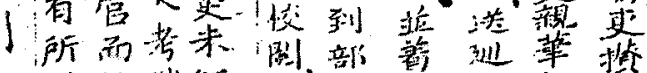

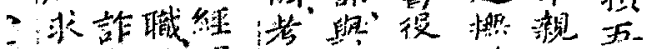

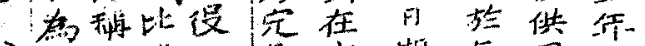

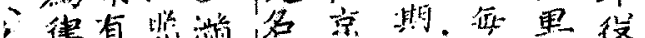

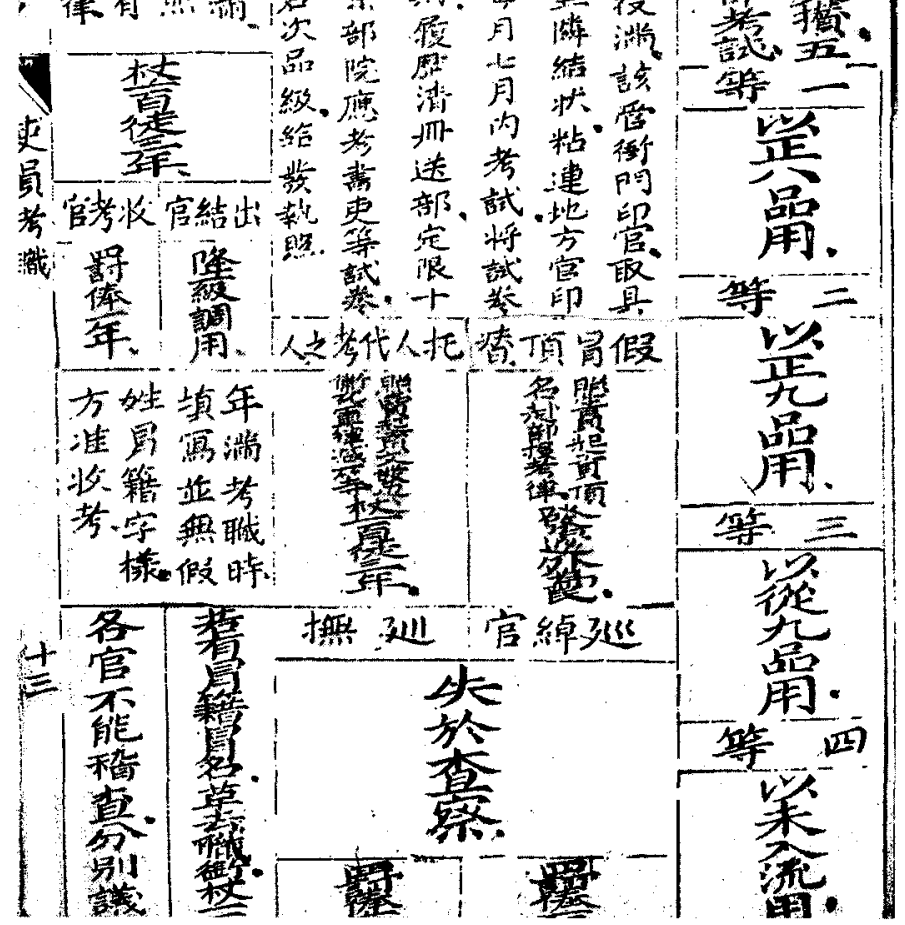

figure 6 
Réglementation administrative et code pénal mis en tableaux

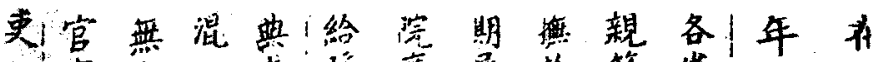

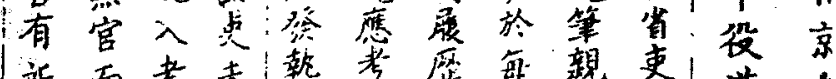

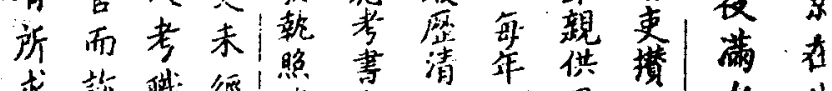

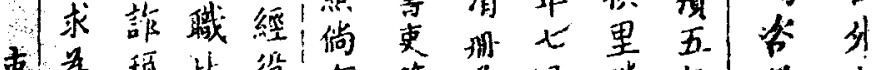

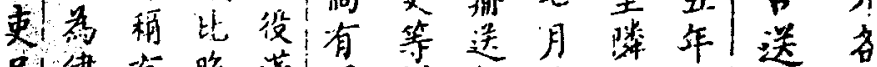

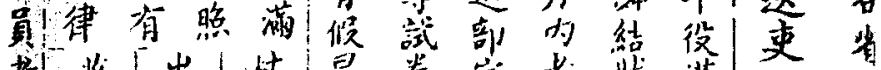

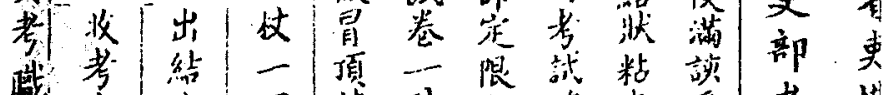
瞳考 絬

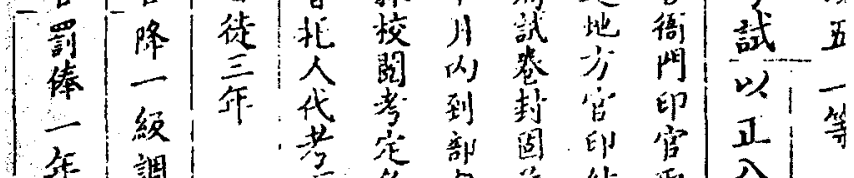

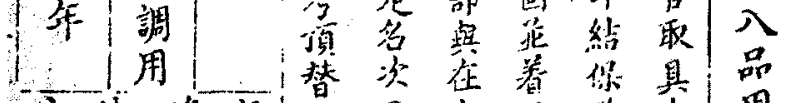

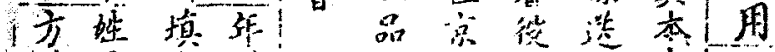

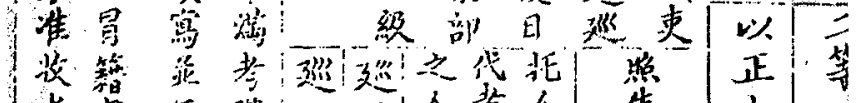

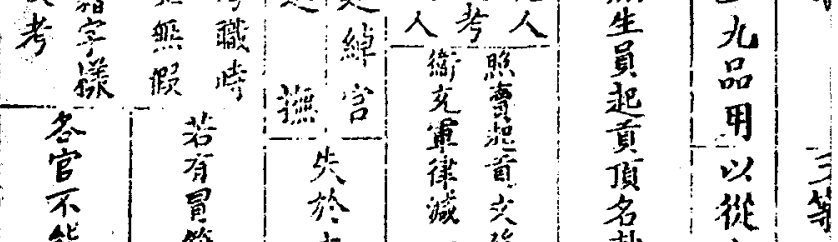

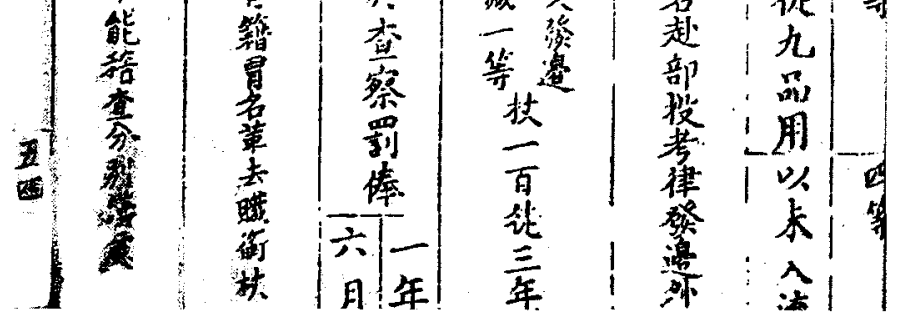

figure 7 


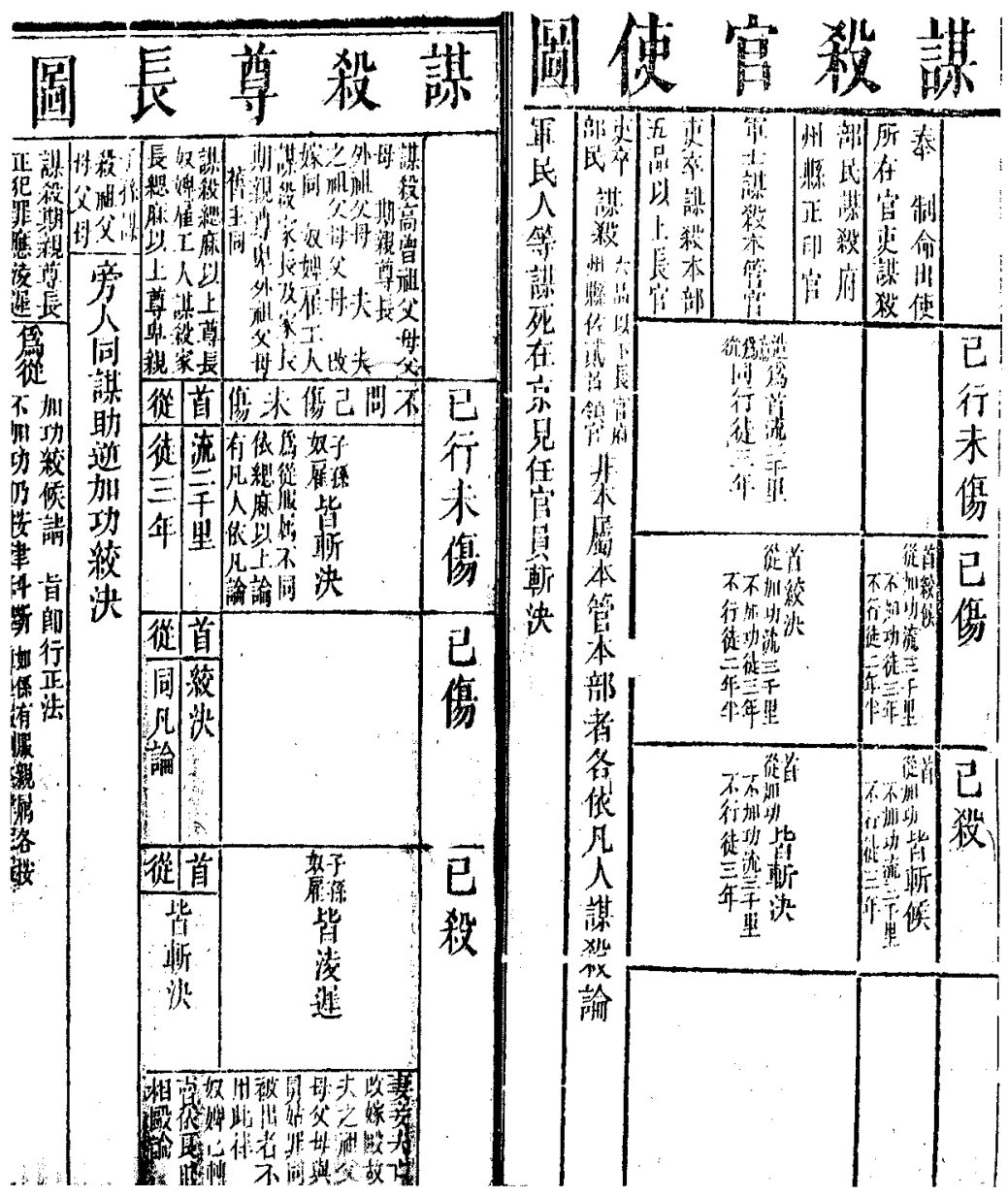

figure 8 


\title{
Réglementation administrative et code pénal mis en tableaux
}

\begin{abstract}
Résumé
Pierre-Étienne WILL : La réglementation administrative et le code pénal mis en tableaux.

Cet article est consacré à une catégorie particulière de manuels pour fonctionnaires, apparue vers 1730 , où la matière du Code pénal et des règlements sur les «sanctions administratives » (chufen) est présentée sous forme de tableaux plus ou moins complexes. L'objectif était de faciliter l'accès des administrateurs à une législation foisonnante et en constant renouvellement. La première partie fait l'historique du genre à travers la description d'une série de titres dont certains ont été révisés et réédités jusqu'à la fin du $\mathrm{XIX}^{\mathrm{e}}$ siècle. Au passage sont évoqués le milieu de spécialistes dont étaient issus les auteurs, les éditeurs et leurs pratiques, et le public visé. La seconde partie revient plus en détail, et à partir d'exemples précis, sur la logique de la présentation, autrement dit sur les différentes façons dont les tableaux résumaient et réorganisaient les recueils législatifs de façon à les rendre «clairs au premier coup d'œil ».
\end{abstract}

\begin{abstract}
Pierre-Étienne WILL : Administrative rules and and the Penal Code set in table form.

This essay is devoted to a particular type of official handbooks that emerged around 1730 , in which the contents of the Penal Code and of the regulations on "administrative sanctions" (chufen) were set in tables of varying complexity. The aim was to facilitate access to a corpus of laws that was both proliferating and constantly updated. Part One proposes a history of the genre by describing a series of works some of which were revised and reprinted through the end of the nineteenth century. The milieu of administrative specialists that produced the authors, the practices of the publishers, and the targeted public are discussed along the way. Part Two discusses in more detail the logic of table presentation, in other words the various methods employed to abstract and reorganize the text of the legislative compendia so as to make them "plain at first glance".
\end{abstract}

